\title{
A Generally Covariant Theory of Quantized Real Klein-Gordon Field in de Sitter Spacetime
}

\author{
Sze-Shiang Feng \\ Independent Researcher, 9521 Windwood Point, Dayton, OH 45458, USA
}

\begin{abstract}
$\overline{\text { Abstract }}$
We propose in this paper a quantization scheme for the real Klein-Gordon field in de Sitter spacetime. Our scheme is generally covariant with the help of vierbein, which is necessary usually for the spinor field in curved spacetime. We first present a Hamiltonian structure and then quantize the field following the standard approach. For the free field, the time-dependent quantized Hamiltonian is diagonalized by Bogliubov transformation, and the eigenstates at each instant are interpreted as the observed particle states at that instant. The interpretation is supported by the known cosmological redshift formula and the onshell condition of 4-momentum for a free field. Though mathematics is carried out in terms of conformal coordinates for the sake of convenience, the whole theory can be transformed into any other coordinates based on general covariance. It is concluded that particle states, such as vacuum states in particular, are time-dependent and vacuum states at one time evolve into nonvacuum states at later times. The formalism of perturbation is provided with en extended Dirac picture.
\end{abstract}

Keywords: Quantization, Klein-Gordon, de Sitter DOI: 10.31526/LHEP.2020.162

\section{INTRODUCTION}

Is it necessary to pursue a quantum field theory conforming to the principle of general covariance and why should another discussion be on the same topic? To the first question, the answer seems affirmative. To answer the second, one needs to evaluate lots of schemes that have been proposed as of today. It seems to the present author that most of the discussions are not as physically fruitful as conventional quantum field theories, though various mathematical structures have been revealed. And the topic of quantized scalar field in curved spacetime deserves revisits.

A unified theory that can explain all forces in nature in quantized fashion has been a Holy Grail in physics for quite long a time ever since Einstein. Yet, definite progress in relating to the real world is still called for considering the developments as of today in superstring/brane theories. Instead of seeking a final theory in which all fields in nature are quantized, a less ambitious endeavor has been paid to the quantization of all fields except for gravity in curved spacetime, a branch that has been one of the major foci in theoretical physics for decades [1]-[4]. To understand the necessity of quantization of matter fields in curved spacetime, one can consider a basic question: pions in cosmic rays come down to the earth all the way from distant universe, are they quantized particles when they pass some region which may be strongly curved by gravity? The answer is seemingly affirmative; i.e., we should have a complete theory of quantum field theory in curved spacetime. Due to the curvature of spacetime, the canonical quantization of fields is not generally as applicable in curved spacetime as in Minkowski spacetime. Because of this, quantization is implemented in many cases by mode expansion directly and by- passes the discussion of canonical structures. This inevitably entails the difficulty of interpretation of concepts such as particles and vacuum states. Henceforth, observable quantities such as energy and momentum are not clearly defined as in conventional Minkowski spacetime quantum field theories. Since we can choose any coordinate system and obtain a different set of mode solutions, we need to verify the general covariance as required by general relativity itself. As in the quantization of Yang-Mills fields, one can work in different gauge conditions. But the whole framework should prove to be gaugeindependent at the end of the day. Yet, general covariance is either not proved or neglected in various quantization schemes of matter fields in curved spacetime.

Another important issue in the quantization of matter fields in curved spacetime is the specification of Fock space which represents states of quantized particles. In conventional quantum field theories in Minkowski spacetime, one of the axioms of the LSZ framework of quantum field theory (QFT) is $P_{a}|0\rangle=$ 0 [5] where $P_{a}$ is the total energy-momentum operator. Without this condition, we can draw absurd conclusions. Suppose that we have Heisenberg algebra $\left[a, a^{\dagger}\right]=1$; we can have infinite number of ways of implementing Bogliubov transformations like $a=u \alpha+v \beta^{\dagger}$ and $a^{\dagger}=u^{*} \alpha^{\dagger}+v^{*} \beta$ with $|u|^{2}-|v|^{2}=1$. But for a quadratic Hamiltonian, only one transformation can diagonalize the Hamiltonian, and the observed energy quanta are represented by the creation/annihilation operators. Just as in the BCS theory of superconductivity, the basic observed quanta are the quasi-particles. Nevertheless, various approaches to the quantization scheme of matter fields in curved spacetime lack or neglict the Hamiltonian.

We propose in this paper a quantization scheme for real Klein-Gordon field in de Sitter spacetime. Quantum field theories in de Sitter spacetimes have been discussed in various ways (for a survey, see [6]). To the present author, the merit lies in a number of aspects. First, Minkowski spacetime is not a so- 
lution to the Einstein equation in the presence of non-vanishing cosmological constant, and the simplest solution is de Sitter spacetime. Second, de Sitter spacetime is a maximally symmetric spacetime as is Minkowski spacetime. Though it is widely recognized that discussions regarding Dirac field in curved spacetime require vierbein representing the gravitational field, we here also introduce vierbein even for the quantization of Klein-Gordon field which is a scalar. The rationale behind is threefold. First, it is necessary even in quantizing a 1D mechanical system. Consider a system of $H=(1 / 2 m) a^{2}(s) \dot{q}^{2}(s)+$ $V(q)$. By redefining time variable as $t=\int a^{-1}(s) d s$, the rest of the procedure becomes standard. Though this example seems trivial, it indicates that vierbein seems indispensable in quantizing systems in curved spacetime. Second, we intend to put the quantization of both scalar fields and spinor fields on the same footing and keep the whole framework coordinate independent. Third, we believe that quantization is about physical observables, as Heisenberg had realized some nine decades before (a historical account for Heisenberg's original thought is provided in [7]). As is well known, Noether's theorem reveals the intrinsic relations between conservation laws and symmetry/invariances of the physical system under consideration. Energy-momentum conservation corresponds to invariance under spacetime translation whereas angular-momentum conservation corresponds to invariance under spacetime rotation. Yet, for general curved coordinate $x^{\mu}$, the invariance under transformation with $\delta x^{\mu}=$ Const does not correspond to translation in general since it might realize a rotation should it be an angular coordinate. Nevertheless, $\delta x^{\mu}=e_{a}^{\mu} b^{a}$ with $b^{a}=$ Const always represents a local spacetime translation since the projection $\delta x^{\mu} e_{\mu}^{a}$ of $\delta x^{\mu}$ on local frame $e_{\mu}^{a}$ is a translation. This argument led to a generally covariant formulation of energy-momentum conservation of matter-gravitation system [8], a quintessential example showing the significance of vierbein in general relativity. As has been discussed in [9]-[12], observed time and space intervals are projections of coordinate intervals onto the local Lorentz frame of the observer. The varying rate of a field in space and time should be measured over the observed space and time instead of the coordinates. Hence, vierbein is essentially necessary for discussions of all kinds of fields.

Discussions of quantum mechanics in de Sitter spacetime were initiated shortly after the birth of the relativistic mechanics of electrons [13]-[22] and have been long since an important topic of quantum theories in curved spacetime. Quantum field theories have been formulated in different approaches and different coordinate systems. Group-theoretic approaches to quantum field theories in de Sitter spacetime are proposed in [23]- [25]. Since de Sitter spacetime can be imbedded in 1+4 dimensional pseudo-Euclidean spacetime, quantum field theories have been formulated in terms of $1+4$ pseudo-Euclidean coordinates [26]-[27], spherical coordinates [28]-[29], static coordinate [30]-[36], and comoving coordinates [37]-[48]. Unlike standard quantum field theory in the Minkowski spacetime which is the Lorentz invariant, most of these theories have not either general covariance or important concepts such as Hamil- tonian and measurable particle states. In our present paper, we seek a generally invariant formalism for quantization and develop physical concepts such as particles and vacuum.

Our goals of this paper are threefold: (i) providing a generally covariant quantum theory of Klein-Gordon field in de Sitter spacetime, in light of the fact that existing theories have not general covariance either implicitly or explicitly, (ii) providing observable quantities of the field quanta, and (iii) providing calculation approaches for scattering matrix.

The present paper is arranged as follows. In Section 2, we present the canonical structure of a real Klein-Gordon field in de Sitter spacetime following the standard approach. Upon redefining canonical variables, the Hamiltonian equations of motion of canonical momentum to the field are simplified. In Section 3, the system is quantized in Schrödinger picture. Section 4 is a review of fundamental solutions of Klein-Gordon field in de Sitter spacetime, as a preparation of second quantization. Section 5 presents in detail the field 2nd quantization and the quantized Hamiltonian both in Heisenberg picture and in Schrödinger picture. The Hamiltonians are diagonalized and quasi-particle creation/annihilation operators are defined. Discussions of difference as well as things in common in the two pictures are provided. Time-dependent vacuum and particles states are defined. Particularly, the observed energymomentum is obtained based on our previous work, and the on-shell relation for free particles is obtained. Some simple matrix elements are calculated. In Section 6, we define a generating function which can be used to calculate various matrix elements. In Section 7, transition amplitude between the two states at different times is formulated. Section 8 is devoted to the formulation of perturbation theory for the interacting field, with the help of the Dirac picture. Section 9 is a conclusional discussion and prospect of this work.

\section{CANONICAL QUANTIZATION OF REAL KLEIN-GORDON FIELD STATIONARY SPACETIME}

\section{1. de Sitter Spacetime}

As a special case of Robertson-Walker spacetime, the de Sitter spacetime is most easily represented as the hyperboloid [1]

$$
\eta_{A B} z^{A} z^{B}=\left(z^{0}\right)^{2}-\left(z^{1}\right)^{2}-\left(z^{2}\right)^{2}-\left(z^{3}\right)^{2}-\left(z^{4}\right)^{2}=-\ell^{2}
$$

embedded in 5-dimensional Minkowski space with metric

$$
d s_{5}^{2}=\left(d z^{0}\right)^{2}-\left(d z^{1}\right)^{2}-\left(d z^{2}\right)^{2}-\left(d z^{3}\right)^{2}-\left(d z^{4}\right)^{2} .
$$

(The relation between the parameter $\ell$ and the cosmological constant $\Lambda$ is $\ell=\sqrt{3 / \Lambda}$.) We choose the coordinates $(t, x)$ defined by

$$
\left\{\begin{array}{c}
z^{0}=\ell \sinh \frac{t}{\ell}+\frac{1}{2 \ell} e^{t / \ell}|x|^{2} \\
z^{4}=\ell \cosh \frac{t}{\ell}-\frac{1}{2 \ell} e^{t / \ell}|x|^{2} \\
z^{i}=e^{t / \ell} x^{i} .
\end{array}\right.
$$

We consider the range covered by $0 \leq t<+\infty,-\infty<x^{i}<\infty$ since we take $t=0$ as the inception of the evolution of the 
universe. The induced line element on the hyperboloid is

$$
d s^{2}=d t^{2}-e^{2 t / \ell} \sum_{i=1}^{3}\left(d x^{i}\right)^{2} .
$$

Now, we define a conformal time

$$
\zeta=\ell e^{-t / \ell}, \quad 0<\zeta<\ell,
$$

and then

$$
d s^{2}=C(\zeta)\left[d \zeta^{2}-\sum_{i}\left(d x^{i}\right)^{2}\right]
$$

with conformal factor $C(\zeta)=(\ell / \zeta)^{2}$. We use $x=(\zeta, x)$ hereinafter.

\subsection{Hamiltonian Structure}

We use the standard definition of vierbein $g_{\mu v}=\eta_{a b} e_{\mu}^{a} e_{b}^{v}$. In the present paper, $e$ sometimes denotes vierbein, it sometimes denotes $e=\sqrt{-g}$, and it sometimes denotes the base of natural exponential, depending on the contexts. Denoting that $\hat{\nabla}_{a}=$ $e_{a}^{\mu} \nabla_{\mu}$ (here $e_{a}^{\mu}$ plays the role of parameter $\lambda(t)$ in [49]) and $\nabla_{\mu}$ is the standard covariant derivative, Lagrange of a free real KleinGordon field is

$$
\begin{aligned}
\mathscr{L} & =\frac{1}{2}\left(\hat{\nabla}^{a} \phi \hat{\nabla}_{a} \phi-m_{\mathrm{KG}}^{2} \phi^{2}\right) \\
& =\frac{1}{2}\left(\hat{\nabla}^{0} \phi \hat{\nabla}_{0} \phi+\hat{\nabla}^{a^{\prime}} \phi \hat{\nabla}_{a^{\prime}} \phi-m_{\mathrm{KG}}^{2} \phi^{2}\right) .
\end{aligned}
$$

Defining [50]

$$
\Pi=\frac{\partial \mathscr{L}}{\partial\left(\hat{\nabla}_{0} \phi\right)}
$$

and assuming the existence of the inverse (for our case, it is apparent)

$$
\hat{\nabla}_{0} \phi=\hat{\nabla}_{0} \phi\left(\phi, \hat{\nabla}_{i} \phi, \Pi ; x\right),
$$

the Hamiltonian is defined in the standard way

$$
\mathscr{H}=\hat{\nabla}_{0} \phi \cdot \Pi-\mathscr{L} .
$$

Thus,

$$
\begin{gathered}
\frac{\partial \mathscr{H}}{\partial \Pi}=\hat{\nabla}_{0} \phi+\Pi \frac{\partial\left(\hat{\nabla}_{0} \phi\right)}{\partial \Pi}-\frac{\partial \mathscr{L}}{\partial\left(\hat{\nabla}_{0} \phi\right)} \frac{\partial\left(\hat{\nabla}_{0} \phi\right)}{\partial \Pi}=\hat{\nabla}_{0} \phi, \\
\frac{\partial \mathscr{H}}{\partial \phi}=\Pi \frac{\partial\left(\hat{\nabla}_{0} \phi\right)}{\partial \phi}-\frac{\partial \mathscr{L}}{\partial\left(\hat{\nabla}_{0} \phi\right)} \frac{\partial\left(\hat{\nabla}_{0} \phi\right)}{\partial \phi}-\frac{\partial \mathscr{L}}{\partial \phi}=-\frac{\partial \mathscr{L}}{\partial \phi} .
\end{gathered}
$$

Using the Euler-Lagrange equation, we have

$$
\frac{\partial \mathscr{L}}{\partial \phi}=\nabla_{\mu}\left[e_{0}^{\mu} \frac{\partial \mathscr{L}}{\partial\left(\hat{\nabla}_{0} \phi\right)}\right]+\nabla_{\mu}\left[e_{a^{\prime}}^{\mu} \frac{\partial \mathscr{L}}{\partial\left(\hat{\nabla}_{a^{\prime}} \phi\right)}\right]
$$

(here, the prime indices such as $a^{\prime}$ run through $1,2,3$ ) and we have

$$
\begin{aligned}
\frac{\partial \mathscr{H}}{\partial \phi} & =-\nabla_{\mu} \frac{\partial \mathscr{L}}{\partial \nabla_{\mu} \phi} \\
& =-\nabla_{\mu}\left[e_{0}^{\mu} \frac{\partial \mathscr{L}}{\partial\left(\hat{\nabla}_{0} \phi\right)}\right]-\nabla_{\mu}\left[e_{a^{\prime}}^{\mu} \frac{\partial \mathscr{L}}{\partial\left(\hat{\nabla}_{a^{\prime}} \phi\right)}\right] \\
& =-\nabla_{\mu}\left[e_{0}^{\mu} \Pi\right]-\nabla_{\mu}\left[e_{a^{\prime}}^{\mu} \frac{\partial \mathscr{L}}{\partial\left(\hat{\nabla}_{a^{\prime}} \phi\right)}\right] .
\end{aligned}
$$

Since

$$
\begin{aligned}
\frac{\partial \mathscr{H}}{\partial\left(\hat{\nabla}_{a^{\prime}} \phi\right)} & =\Pi \frac{\partial\left(\hat{\nabla}_{0} \phi\right)}{\partial\left(\hat{\nabla}_{a^{\prime}} \phi\right)}-\frac{\partial \mathscr{L}}{\partial\left(\hat{\nabla}_{0} \phi\right)} \frac{\partial\left(\hat{\nabla}_{0} \phi\right)}{\partial\left(\hat{\nabla}_{a^{\prime}} \phi\right)}-\frac{\partial \mathscr{L}}{\partial\left(\hat{\nabla}_{a^{\prime}} \phi\right)} \\
& =-\frac{\partial \mathscr{L}}{\partial\left(\hat{\nabla}_{a^{\prime}} \phi\right)},
\end{aligned}
$$

we find

$$
\nabla_{\mu}\left[e_{0}^{\mu} \Pi\right]=-\frac{\partial \mathscr{H}}{\partial \phi}+\nabla_{\mu}\left[e_{a^{\prime}}^{\mu} \frac{\partial \mathscr{H}}{\partial\left(\hat{\nabla}_{a^{\prime}} \phi\right)}\right]
$$

Introducing the functional derivative

$$
\frac{\delta}{\delta \psi}=\frac{\partial}{\partial \psi}-\nabla_{\mu}\left[e_{a^{\prime}}^{\mu} \frac{\partial}{\partial\left(\hat{\nabla}_{a^{\prime}} \psi\right)}\right],
$$

we have

$$
\begin{gathered}
\hat{\nabla}_{0} \phi=\frac{\delta \mathscr{H}}{\delta \Pi}, \\
\nabla_{\mu}\left[e_{0}^{\mu} \Pi\right]=-\frac{\delta \mathscr{H}}{\delta \phi} .
\end{gathered}
$$

These can be cast into the conventional formalism. Defining Cauchy surface $\Sigma: f(x)=\zeta=$ const, we have the functional

$$
H[\phi, \Pi ; \zeta]:=\int_{\Sigma} d \sigma \mathscr{H},
$$

where

$$
\begin{aligned}
\mathscr{H} & =\frac{1}{2}\left(\Pi^{2}-\hat{\nabla}^{a^{\prime}} \phi \hat{\nabla}_{a^{\prime}} \phi+m_{\mathrm{KG}}^{2} \phi^{2}\right), \\
d \sigma_{\mid \Sigma} & :=d \sigma_{\mu} n^{\mu}=\frac{1}{3 !} e \varepsilon_{\mu v \alpha \beta} d x^{v} \wedge d x^{\alpha} \wedge d x^{\beta} n^{\mu} \\
& =C^{3 / 2}(\zeta) d^{3} x .
\end{aligned}
$$

Here, $n^{\mu}=C^{-1 / 2}(\zeta)(1,0,0,0)$ is the unit normal to $\Sigma$. For two functionals $F[\phi, \Pi ; \zeta]=\int_{\Sigma} d \sigma \mathscr{F}$ and $G[\phi, \Pi ; \zeta]=\int_{\Sigma} d \sigma \mathscr{G}$, the Poisson bracket is defined as

$$
\{F, G\}^{\text {P.B. }}:=\int_{\Sigma} d \sigma\left(\frac{\delta \mathscr{F}}{\delta \phi(x)} \frac{\delta \mathscr{G}}{\delta \Pi(x)}-\frac{\delta \mathscr{G}}{\delta \phi(x)} \frac{\delta \mathscr{F}}{\delta \Pi(x)}\right) .
$$

Since $\phi(x)=\int_{\Sigma} d \sigma^{\prime} \delta^{3}\left(x-x^{\prime}\right) C^{1 / 2}(\zeta) \phi\left(x^{\prime}\right)$ and $\Pi(y)=$ $\int_{\Sigma} d \sigma^{\prime} \delta^{3}\left(y-y^{\prime}\right) C^{1 / 2}(\zeta) \Pi\left(y^{\prime}\right)$, we have then

$$
\{\phi(x), \Pi(y)\}^{\text {P.B. }}=\delta^{3}\left(Z^{i}\right)=\frac{1}{e^{\prime}} \delta^{3}(x-y) .
$$

Here, $Z^{i}$ are the local flat coordinates defined as $Z^{i}=e_{\mu}^{i}(x-$ $y)^{\mu}$ for $y$ being very close to $x$ and $e^{\prime}=\operatorname{det}\left(e_{\mu^{\prime}}^{a^{\prime}}\right)$. Hence, we have

$$
\begin{aligned}
\hat{\nabla}_{0} \phi & =\{\phi(x), H\}_{x^{0}=\zeta}^{\text {P.B. }}, \\
\hat{\nabla}_{0} \Pi & =\{\Pi(x), H\}_{x^{0}=\zeta}^{\text {P.B. }}-\omega_{0} \Pi,
\end{aligned}
$$

where

$$
\omega_{0}=\nabla_{\mu} e_{0}^{\mu}=\frac{1}{C^{2}(\zeta)} \partial_{\zeta}\left(C^{2}(\zeta) C^{-1 / 2}(\zeta)\right)
$$




\subsection{Appropriate Phase Space Variables}

Since the equation for $\Pi$ involves a term $\omega_{0} \Pi$, which does not exist in conventional canonical equations of motion, we seek new definitions of canonical variables. Defining $\pi:=e^{\Omega} \Pi$ where $\hat{\nabla}_{0} \Omega=\omega_{0}$ which is a scalar, we have $\Omega=\ln C^{3 / 2}(\zeta)$ and then

$$
\begin{aligned}
e_{0}^{\mu} \nabla_{\mu} \pi & =e^{\Omega} \hat{\nabla}_{0} \Pi+\hat{\nabla}_{0} e^{\Omega} e^{\Omega} \Pi=e^{\Omega} \hat{\nabla}_{0} \Pi+\hat{\nabla}_{0} \Omega \cdot \pi \\
& =e^{\Omega}\left(\{\Pi(x), H\}_{x^{0}=\zeta}^{\text {P.B. }}-\omega_{0} \Pi\right)+\omega_{0} \cdot \pi \\
& =\{\pi(x), H\}_{x^{0}=\zeta}^{\text {P.B. }} .
\end{aligned}
$$

In terms of $\phi, \pi$, we have

$$
\begin{gathered}
\hat{\nabla}_{0} \phi=\{\phi(x), H[\phi, \pi ; \zeta]\}_{x^{0}=\zeta}^{\text {P.B. }}, \\
\hat{\nabla}_{0} \pi=\{\pi(x), H[\phi, \pi ; \zeta]\}_{x^{0}=\zeta}^{\text {P.B. }}, \\
\{\phi(x), \pi(y)\}^{\text {P.B. }}=e^{\Omega} \delta^{3}\left(Z^{i}\right)=\delta^{3}(\boldsymbol{x}-\boldsymbol{y}) .
\end{gathered}
$$

It is worthwhile to point out the two benefits of using $\pi$ instead of $\Pi$. First, the classical equation of $\pi$ does not have the term $\omega_{0} \Pi$ on the right. Second, the Poisson bracket equation (31) does not depend on time, a nice feature when we transform from Heisenberg picture to Schrödinger picture later on. In Schrödinger picture, canonical conjugate fields do not depend on time and hence should not do their commutators.

In general, for any $O[\phi, \pi ; \lambda(x)]$,

$$
O[\phi, \pi ; \lambda(x)]=\int_{\Sigma} d \sigma \mathscr{O}(\phi, \pi ; \lambda(x))
$$

which is a function of $\zeta$ and a function of $\phi$ and $\pi$

$$
\begin{aligned}
\hat{d}_{0} O[\phi, \pi ; \lambda(x)](\zeta) & =\int_{\Sigma} e_{0}^{\zeta}(x) \partial_{\zeta}(d \sigma) \mathscr{O}+\{O, H\} \\
& +\int_{\Sigma} d \sigma\left(-\frac{\delta \mathscr{O}}{\delta \pi(x)} \omega_{0} \pi+\frac{\delta \mathscr{O}}{\delta \lambda(x)} \hat{\nabla}_{0} \lambda\right)
\end{aligned}
$$

where $\hat{d}_{0}$ is defined as $e_{0}^{\zeta}(\zeta) d / d \zeta$, bearing in mind that $\zeta=$ const. defines the surface $\Sigma$; i.e., the l.h.s. depends on the surface $\Sigma$. In particular,

$$
\begin{aligned}
\hat{d}_{0} H[\phi, \pi ; \cdots]= & \int_{\Sigma} e_{0}^{\zeta}(x) \partial_{\zeta}(d \sigma) \mathscr{H} \\
& -\int_{\Sigma} d \sigma\left(\frac{\delta \mathscr{H}}{\delta \pi(x)} \omega_{0} \pi+\frac{\delta \mathscr{H}}{\delta e_{a}^{\mu}} \hat{\nabla}_{0} e_{a}^{\mu}\right) .
\end{aligned}
$$

In terms of canonical variables $\phi$ and $\pi$

$$
\mathscr{H}=\frac{1}{2}\left(e^{-2 \Omega} \pi^{2}-\hat{\nabla}^{a^{\prime}} \phi \hat{\nabla}_{a^{\prime}} \phi+m_{\mathrm{KG}}^{2} \phi^{2}\right) .
$$

Defining the new Poisson bracket

$$
\{F, G\}^{\text {new }}:=\int_{\Sigma} d \sigma e^{\Omega(x)}\left(\frac{\delta \mathscr{F}}{\delta \phi(x)} \frac{\delta \mathscr{G}}{\delta \pi(x)}-\frac{\delta \mathscr{G}}{\delta \phi(x)} \frac{\delta \mathscr{F}}{\delta \pi(x)}\right),
$$

then,

$$
\begin{aligned}
\hat{\nabla}_{0} \phi & =\{\phi(x), H[\phi, \pi ; \zeta]\}_{x^{0}=\zeta}^{\text {new }}, \\
\hat{\nabla}_{0} \pi & =\{\tilde{\Pi}(x), H[\phi, \pi ; \zeta]\}_{x^{0}=\zeta}^{\text {new }}
\end{aligned}
$$

\section{SCHRÖDINGER PICTURE}

Upon quantization, the classical canonical variables $\phi$ and $\pi$ are replaced by the operators $\hat{\phi}$ and $\hat{\pi}$ in a Hilbert space and Poisson brackets become commutators. In standard quantized Klein-Gordon field theory in Minkowski spacetime, the Hamiltonian is time-independent and three pictures can be utilized. Similarly, we can define Schrödinger picture $O=\phi, \pi$ :

$$
O^{\mathrm{S}}(\zeta)=\hat{T}^{-1} e^{i \int_{\ell}^{\zeta} H(\eta) e_{\zeta}^{0}(\eta) d \eta} O(\zeta) \hat{T} e^{-i \int_{\ell}^{\zeta} H(\eta) e_{\zeta}^{0}(\eta) d \eta},
$$

where $\hat{T}$ is the time-ordering operator defined as $\hat{T} \phi\left(\zeta_{1}\right) \phi\left(\zeta_{2}\right)=$ $\phi\left(\zeta_{1}\right) \phi\left(\zeta_{2}\right) \theta\left(\zeta_{2}-\zeta_{1}\right)+\phi\left(\zeta_{2}\right) \phi\left(\zeta_{1}\right) \theta\left(\zeta_{1}-\zeta_{2}\right)$. The two pictures agree at $\zeta=\ell$ :

$$
\phi^{\mathrm{S}}(x)=\phi(\ell, x)
$$

For Hamiltonian,

$$
\begin{aligned}
H^{\mathrm{S}}(\zeta) & =\hat{T}^{-1} e^{i \int_{\ell}^{\zeta} H(\eta) e_{\zeta}^{0}(\eta) d \eta} H(\zeta) \hat{T} e^{-i \int_{\ell}^{\zeta} H(\eta) e_{\zeta}^{0}(\eta) d \eta} \\
& =H\left[\phi^{\mathrm{S}}(\boldsymbol{x}), \pi^{\mathrm{S}}(\boldsymbol{x}) ; e_{a}^{\mu}(x), \Omega(x)\right](\zeta)
\end{aligned}
$$

and $H^{\mathrm{S}}(\ell)=H(\ell)$. Since

$$
i \hat{\nabla}_{0} \phi^{\mathrm{S}}(x)=0,
$$

$\phi^{\mathrm{S}}$ and $\pi^{\mathrm{S}}$ are time-independent, playing the roles of $x$ and $p$ while $\phi$ and $\pi$ play the role $x_{ \pm}, p_{ \pm}$in [59] :

$$
\begin{gathered}
H(t)=f(t) p^{2} / 2 m+g(t) \frac{1}{2} m \omega_{0}^{2} x^{2}, \\
H_{+}(t)=f(t) p_{+}^{2}(t) / 2 m+g(t) \frac{1}{2} m \omega_{0}^{2} x_{+}^{2}(t), \\
d H(t) / d t=\dot{f}(t) p^{2} / 2 m+\dot{g}(t) \frac{1}{2} m \omega_{0}^{2} x^{2}, \\
d H_{+}(t) / d t=\dot{f}(t) p_{+}^{2}(t) / 2 m+\dot{g}(t) \frac{1}{2} m \omega_{0}^{2} x_{+}^{2}(t) .
\end{gathered}
$$

Though the initial condition $H(0)=H_{+}(0)$, yet $H(t) \neq H_{+}(t)$, since $p_{+}(t)$ and $x_{+}(t)$ depend on time $t$. The time dependence of $H^{S}$ is

$$
i \hat{d}_{0} H^{\mathrm{S}}(\zeta)=i e_{0}^{\zeta} \frac{\partial}{\partial \zeta} H\left[\phi^{\mathrm{S}}(x), \pi^{\mathrm{S}}(x) ; e_{a}^{\mu}(x), \Omega(x)\right](\zeta) .
$$

Quantization is carried out by the correspondence

$$
[\hat{\phi}(\boldsymbol{x}), \hat{\pi}(\boldsymbol{y})]_{x^{0}=y^{0}}:=i \hbar\{\phi(x), \pi(y)\}_{x^{0}=y^{0}}^{\text {new }}=i \hbar \delta^{3}(\boldsymbol{x}-\boldsymbol{y}) .
$$

Quantum mechanical Schrödinger equation for the wave functional

$$
i \hat{\nabla}_{0} \Psi[\phi(x), \zeta]=H^{S}[\phi(x), \pi(x) ; \zeta] \Psi[\phi(x), \zeta],
$$

where in $H^{S}$ (as in conventional quantum field theories, this is not unique!) $\pi(\boldsymbol{x}) \mapsto-i \hbar \delta / \delta \phi(\boldsymbol{x})$. So,

$$
\begin{aligned}
& H^{S}(\zeta) \\
= & \left.\frac{1}{2} \int_{\Sigma} d \sigma\left[e^{-2 \Omega} \pi^{2}(\boldsymbol{x})-\hat{\nabla}^{a^{\prime}} \phi(\boldsymbol{x}) \hat{\nabla}_{a^{\prime}} \phi(\boldsymbol{x})+m_{\mathrm{KG}}^{2} \phi^{2}(\boldsymbol{x})\right)\right] \\
= & \frac{1}{2} \int_{\Sigma} d \sigma\left[C^{-3}(\zeta)\left(-i \hbar \frac{\delta}{\delta \phi(\boldsymbol{x})}\right)^{2}-\hat{\nabla}^{a^{\prime}} \phi(\boldsymbol{x}) \hat{\nabla}_{a^{\prime}} \phi(\boldsymbol{x})\right. \\
& \left.+m_{\mathrm{KG}}^{2} \phi^{2}(\boldsymbol{x})\right] .
\end{aligned}
$$


This is slightly different from [61]. The Gaussian-type Schrödinger with wave functional $\Psi[\phi(\boldsymbol{x}), \zeta]=\eta e^{-G[\phi(x), \zeta]}$ is satisfied by equation (46):

$$
\begin{aligned}
& H^{\mathrm{S}}[\phi(\boldsymbol{x}), \pi(\boldsymbol{x}) ; \zeta] \Psi[\phi(\boldsymbol{x}), \zeta] \\
= & \frac{1}{2} \int d \sigma\left[C^{-3}(\zeta)\left(\frac{\delta^{2} G}{\delta \phi(\boldsymbol{x})^{2}}-\left(\frac{\delta G}{\delta \phi(\boldsymbol{x})}\right)^{2}\right)\right. \\
& \left.-\hat{\nabla}^{a^{\prime}} \phi \hat{\nabla}_{a^{\prime}} \phi+m_{\mathrm{KG}}^{2} \phi^{2}\right] \Psi[\phi(\boldsymbol{x}), \zeta] .
\end{aligned}
$$

Assuming

$$
G[\phi(x), \zeta]=i E(\zeta)+\int d^{3} x \int d^{3} y \phi(x) f(x, y ; \zeta) \phi(y),
$$

then,

$$
\begin{aligned}
& -2 C^{-3}(\zeta) \int d \sigma d^{3} \boldsymbol{y} d^{3} z \phi(z) f(z, x ; \zeta) f(x, y ; \zeta) \phi(y) \\
& +\frac{1}{2} \int d \sigma \phi(x)\left[e_{a^{\prime}}^{\mu} e_{b^{\prime}}^{v} \eta^{a^{\prime} b^{\prime}} \nabla_{\mu} \nabla_{v}+m_{\mathrm{KG}}^{2}\right] \phi(\boldsymbol{x}) \\
= & i e_{0}^{\zeta}(\zeta) \int d^{3} \boldsymbol{x} \int d^{3} \boldsymbol{y} \phi(\boldsymbol{x}) \partial_{\zeta} f(\boldsymbol{x}, \boldsymbol{y} ; \zeta) \phi(\boldsymbol{y}) .
\end{aligned}
$$

Using the Fourier transformation $f(x, y ; \zeta)=\sum_{k} f_{k}(\zeta) e^{i k \cdot(x-y)}$, we get

$$
-2 C^{-1}(\zeta)(2 \pi)^{3} f_{k}^{2}(\zeta)+\frac{1}{2} C(\zeta)(2 \pi)^{3} \omega_{k}^{2}(\zeta)=i \partial_{\zeta} f_{k}(\zeta)
$$

which is a Riccati equation and can be transformed into a Bessel-type equation.

\section{FUNDAMENTAL SOLUTIONS FOR FREE FIELD}

\subsection{Basis Solutions}

As has been long since known, the equation $\left(\nabla^{2}+m_{\mathrm{KG}}^{2}\right) \phi=0$ in terms of comoving coordinates of de Sitter spacetime reads [1]

$$
C^{-2}(\zeta) \partial_{\zeta}\left(C(\zeta) \partial_{\zeta} \phi\right)+\partial_{i}\left(C^{-1}(\zeta) \eta^{i j} \partial_{j} \phi\right)+m_{\mathrm{KG}}^{2} \phi=0,
$$

and the solutions are $\Phi_{k}=\mathscr{Y}_{k}(x) C^{-1 / 2}(\zeta) f_{k}(\zeta)$, where $\mathscr{Y}_{k}(x)=(2 \pi)^{-3 / 2} e^{i k \cdot x}, \quad \underline{k}=k=\left(k_{1}, k_{2}, k_{3}\right), \quad\left(-\infty<k_{i}<\right.$ $\infty) \cdot f_{\underline{k}}(\zeta)$ satisfies

$$
\ddot{f}_{\underline{k}}(\zeta)+\left[k^{2}+\frac{1}{4}\left(\frac{\dot{C}}{C}\right)^{2}-\frac{1}{2} \frac{\ddot{C}}{C}+m_{\mathrm{KG}}^{2} C(\zeta)\right] f_{\underline{k}}(\zeta)=0 .
$$

For $C(\zeta)=A \zeta^{w}$

$$
\ddot{f}_{\underline{k}}(\zeta)+\left[\boldsymbol{k}^{2}+\frac{1}{4} \frac{w^{2}}{\zeta^{2}}-\frac{1}{2} \frac{w(w-1)}{\zeta^{2}}+m_{\mathrm{KG}}^{2} A \zeta^{w}\right] f_{\underline{k}}(\zeta)=0
$$

In our case $w=-2$, we have

$$
\ddot{f}_{\underline{k}}(\zeta)+\left(k^{2}-\frac{2-\ell^{2} m_{\mathrm{KG}}^{2}}{\zeta^{2}}\right) f_{\underline{k}}(\zeta)=0 .
$$

Let $f_{\underline{k}}(\zeta)=\zeta^{1 / 2} J(\zeta)$; then (where $\left.z=|\boldsymbol{k}| \zeta\right)$,

$$
\ddot{J}(z)+\frac{1}{z} \dot{J}(z)+\left(1-\frac{v^{2}}{z^{2}}\right) J=0
$$

$$
v^{2}=\frac{9}{4}-\ell^{2} m_{\mathrm{KG}}^{2}
$$

(For electron, the Compton wave length $\lambda_{e}=\hbar / m_{e} c=3.86 \times$ $10^{-13} \mathrm{~m}$. So, $\ell / \lambda_{e} \sim 10^{40} \gg$ 1.) Therefore, for $9 / 4>\ell^{2} m_{\mathrm{KG}^{\prime}}^{2}$ (i.e., the complementary series), we have, for $v \neq n$ (denoting $k=|\boldsymbol{k}|)$,

$$
f_{\underline{k}}=\zeta^{1 / 2}\left[\alpha_{1 \underline{k}} H_{v}^{(1)}(k \zeta)+\alpha_{2 \underline{k}} H_{v}^{(2)}(k \zeta)\right]
$$

For integer $v$,

$$
f_{\underline{k}}=\zeta^{1 / 2}\left[\alpha_{1 \underline{k}} J_{n}(k \zeta)+\alpha_{2 \underline{k}} Y_{n}(k \zeta)\right]
$$

Hence, for noninteger $v$ we have

$$
\Phi_{\underline{k}} \sim(2 \pi)^{-3 / 2} e^{i \boldsymbol{k} \cdot x} \frac{\zeta^{3 / 2}}{\ell}\left[\alpha_{1 \underline{k}} H_{v}^{(1)}(k \zeta)+\alpha_{2 \underline{k}} H_{v}^{(2)}(k \zeta)\right] .
$$

As we choose $e^{i(-\omega t+k \cdot x)}$ in the Minkowski spacetime, we write $\Phi_{k}(\zeta, x)=: g_{k}(\zeta) e^{i k \cdot x}$, where

$$
g_{k}(\zeta)=(2 \pi)^{-3 / 2} A(\boldsymbol{k}) \zeta^{3 / 2} H_{v}^{(2)}(k \zeta)
$$

where $A(\boldsymbol{k})$ will be determined later by the normalization of Klein-Gordon product. For $\zeta \rightarrow 0$,

$$
g_{k}(\zeta)=(2 \pi)^{-3 / 2} A(\boldsymbol{k}) \zeta^{3 / 2} H_{v}^{(2)}(k \zeta) \rightarrow \zeta^{3 / 2-v} \sim 0,
$$

which is reminiscent of $\lim _{t \rightarrow \infty} e^{i \omega t} \sim 0$ in the sense RiemannLebesgue Lemma of generalized functions. For $9 / 4<$ $\ell^{2} m_{\mathrm{KG}}^{2}$ (i.e., the principal series), letting $v=i \mu$, we have

$$
f_{\underline{k}}=\zeta^{1 / 2}\left[\beta_{1 \underline{k}} H_{i \mu}^{(1)}(k \zeta)+\beta_{2 \underline{k}} H_{i \mu}^{(2)}(k \zeta)\right] .
$$

In this work, we consider the complementary series only and zero-mass cases can be included.

\subsection{Klein-Gordon Current and Mode Expansion}

Conventionally, the Klein-Gordon current defined as [51]

$$
J_{\left(f_{A}, f_{B}\right)}^{\mu}:=f_{A}^{*}(x) \nabla^{\mu} f_{B}(x)-f_{B}(x) \nabla^{\mu} f_{A}^{*}(x)
$$

is conserved.

$$
\nabla_{\mu} J_{\left(f_{A}, f_{B}\right)}^{\mu}=0
$$

Hence, the quantity

$$
\left(f_{A} \mid f_{B}\right)_{\mathrm{KG}}:=i \int_{\Sigma} d \sigma_{\mu} J_{\left(f_{A}, f_{B}\right)}^{\mu}=i \int_{\Sigma} d \sigma f_{A}^{*}(x) \overleftrightarrow{\Sigma_{n}} f_{B}(x)
$$

is constant over the foliations of surfaces $\Sigma$. We have

$$
\left(f_{A} \mid f_{B}\right)_{\mathrm{KG}}^{*}=\left(f_{B} \mid f_{A}\right)_{\mathrm{KG}} .
$$

Suppose that with coordinates $x$ there are a complete set of solutions $f_{i}$ and $f_{i}^{*}$ to the K-G equation satisfying

$$
\begin{aligned}
& \left(f_{i} \mid f_{j}\right)_{\mathrm{KG}}=-\left(f_{i}^{*} \mid f_{j}^{*}\right)_{\mathrm{KG}}=\delta_{i j}, \\
& \left(f_{i}^{*} \mid f_{j}\right)_{\mathrm{KG}}=\left(f_{i} \mid f_{j}^{*}\right)_{\mathrm{KG}}=0,
\end{aligned}
$$


and

$$
\sum_{i}\left[f_{i}(x) f_{i}^{*}(y)-f_{i}^{*}(x) f_{i}(y)\right]=0 .
$$

Completeness requires that any K-G solution $g(x)$ can be expanded as

$$
g(x)=\sum_{i}\left(g_{i} f_{i}+g_{i}^{*} f_{i}^{*}\right),
$$

where

$$
g_{i}=\left(f_{i} \mid g\right)_{\mathrm{KG}}, \quad g_{i}^{*}=-\left(f_{i}^{*} \mid g\right)_{\mathrm{KG}} .
$$

So, we have a resolution of identity

$$
\left.\sum_{i} \mid f_{i}\right)\left(f_{i}\left|-\sum_{i}\right| f_{i}^{*}\right)\left(f_{i}^{*} \mid=1\right.
$$

or

$$
\begin{aligned}
& i \sum_{i}\left[f_{i}(x)\left(f_{i}^{*}(y) n^{\mu}(y) \nabla_{\mu}-n^{\mu}(y) \nabla_{\mu} f_{i}^{*}(y)\right)\right. \\
& \left.-f_{i}^{*}(x)\left(f_{i}(y) n^{\mu}(y) \nabla_{\mu}-n^{\mu}(y) \nabla_{\mu} f_{i}(y)\right)\right]_{\mid \Sigma}=\delta^{3}\left(Z^{i}\right) .
\end{aligned}
$$

Since if $f_{i}(x)$ is a solution, so must be $f_{i}^{*}(x)$, therefore we have

$$
\begin{aligned}
& \sum_{i}\left[f_{i}(x) n^{\mu}(y) \nabla_{\mu} f_{i}^{*}(y)-f_{i}^{*}(x) n^{\mu}(y) \nabla_{\mu} f_{i}(y)\right]_{\mid \Sigma} \\
& =i \delta^{3}\left(Z^{i}\right) .
\end{aligned}
$$

For free field, we can expand

$$
\phi(x)=\sum_{i}\left[a_{i} f_{i}+a_{i}^{\dagger} f_{i}^{*}\right],
$$

and then

$$
a_{i}=\left(f_{i} \mid \phi\right)_{\mathrm{KG}}, \quad a_{j}^{\dagger}=-\left(f_{j}^{*} \mid \phi\right)_{\mathrm{KG}} .
$$

Since

$$
\begin{aligned}
\left(f_{i} \mid \phi\right)_{\mathrm{KG}} & =i \int_{\Sigma} d \sigma n_{\mu}\left(f_{i}^{*} \nabla^{\mu} \phi-\phi \nabla^{\mu} f_{i}^{*}\right) \\
& =i \int_{\Sigma} d \sigma\left(f_{i}^{*} \Pi-n_{\mu} \phi \nabla^{\mu} f_{i}^{*}\right), \\
\left(f_{j}^{*} \mid \phi\right)_{\mathrm{KG}} & =i \int_{\Sigma} d \sigma n_{\mu}\left(f_{j} \nabla^{\mu} \phi-\phi \nabla^{\mu} f_{j}\right) \\
& =i \int_{\Sigma} d \sigma\left(f_{j} \Pi-n_{\mu} \phi \nabla^{\mu} f_{j}\right),
\end{aligned}
$$

hence,

$$
\begin{gathered}
{\left[a_{i}, a_{j}^{\dagger}\right]=i \int_{\Sigma_{\mathrm{KG}}} d \sigma f_{i}^{*} \overleftrightarrow{L_{n}} f_{j}=\left(f_{i} \mid f_{j}\right)_{\mathrm{KG}}=\delta_{i j},} \\
\Pi(y)=n^{\mu} \sum_{j} \nabla_{\mu}\left(a_{j} f_{j}+a_{j}^{\dagger} f_{j}^{*}\right) \\
{[\phi(x), \Pi(y)]_{\mid \Sigma}} \\
=\sum_{i}\left[f_{i}(x) \nabla_{\mu} f_{i}^{*}(y)-f_{i}^{*}(x) \nabla_{\mu} f_{i}(y)\right] n^{\mu}(y)=i \delta^{3}\left(Z^{i}\right)
\end{gathered}
$$

The K-G product on $\Sigma$ defined by $\zeta=\zeta_{0}$ is

$$
\begin{aligned}
& \left(\Phi_{\boldsymbol{k}} \mid \Phi_{\boldsymbol{k}^{\prime}}\right)_{\mathrm{KG}} \\
= & i \int_{\zeta=\zeta_{0}} d \sigma_{\mu}\left(\Phi_{k}^{*} \nabla^{\mu} \Phi_{\boldsymbol{k}^{\prime}}-\nabla^{\mu} \Phi_{\boldsymbol{k}}^{*} \Phi_{\boldsymbol{k}^{\prime}}\right) \\
= & i \int \delta\left(\zeta-\zeta_{0}\right)\left(\Phi_{k}^{*} \nabla_{\zeta} \Phi_{\boldsymbol{k}^{\prime}}-\nabla_{\zeta} \Phi_{k}^{*} \Phi_{\boldsymbol{k}^{\prime}}\right) C(\zeta) d \zeta d^{3} \boldsymbol{x} .
\end{aligned}
$$

Using the expression of $\Phi_{k}$, we have

$$
\begin{aligned}
& \left(\Phi_{\boldsymbol{k}} \mid \Phi_{\boldsymbol{k}^{\prime}}\right)_{\mathrm{KG}} \\
= & i|A(\boldsymbol{k})|^{2} \delta^{3}\left(\boldsymbol{k}-\boldsymbol{k}^{\prime}\right) \ell^{2} \zeta_{0}\left[H_{v}^{(2) *}\left(k \zeta_{0}\right) \partial_{\zeta_{0}}\left[H_{v}^{(2)}\left(k \zeta_{0}\right)\right]\right. \\
& -\partial_{\left.\zeta_{0}\left[{ }_{0} H_{v}^{(2) *}\left(k \zeta_{0}\right)\right] H_{v}^{(2)}\left(k \zeta_{0}\right)\right] .}
\end{aligned}
$$

For real or imaginary $v$, the Wronskian of any two solutions $Z_{v}^{1}$ and $Z_{v}^{2}$ to Bessel equation satisfies

$$
z W\left[Z_{v}^{1 *}, Z_{v}^{2}\right]=\text { Const. }
$$

So, we must have

$$
z\left[H_{v}^{(2) *}(z) \partial_{z}\left[H_{v}^{(2)}(z)\right]-\partial_{z}\left[H_{v}^{(2) *}(z)\right] H_{v}^{(2)}(z)\right]=\text { Const. }
$$

To find the constant, we can use the asymptotic behavior, $H_{v}^{(2)}(z) \sim \sqrt{2 / \pi z} e^{-i\left(z-\frac{v \pi}{2}-\frac{\pi}{4}\right)}$, and take limit for large $z$; one obtains

$$
\begin{aligned}
& z\left[H_{v}^{(2) *}(z) \partial_{z}\left[H_{v}^{(2)}(z)\right]-\partial_{z}\left[H_{v}^{(2) *}(z)\right] H_{v}^{(2)}(z)\right] \\
= & \frac{-4 i}{\pi} e^{\frac{\pi i}{2}\left(v-v^{*}\right)},
\end{aligned}
$$

and hence

$$
\left(\Phi_{\boldsymbol{k}} \mid \Phi_{\boldsymbol{k}^{\prime}}\right)_{\mathrm{KG}}=|A(\boldsymbol{k})|^{2} \delta^{3}\left(\boldsymbol{k}-\boldsymbol{k}^{\prime}\right) \ell^{2} \frac{4}{\pi} e^{\frac{\pi i}{2}\left(v-v^{*}\right)},
$$

which is apparently $\zeta_{0}$-independent. Therefore, we should choose

$$
A(\boldsymbol{k})=\frac{\sqrt{\pi}}{2 \ell} e^{-\frac{\pi i}{4}\left(v-v^{*}\right)} .
$$

\section{SECOND QUANTIZATION: FREE FIELD}

Here, we use discrete notation for mode expansion:

$$
\begin{aligned}
\phi(x) & =\sum_{k}\left(a_{k} \Phi_{k}+a_{k}^{\dagger} \Phi_{k}^{*}\right), \\
\Pi & =e_{0}^{\mu} \nabla_{\mu} \phi=C^{-1 / 2}(\zeta) \sum_{p}\left(a_{p} \nabla_{\zeta} \Phi_{p}+a_{p}^{\dagger} \nabla_{\zeta} \Phi_{p}^{*}\right) .
\end{aligned}
$$

We can calculate the basic commutator

$$
\begin{aligned}
& {[\phi(x), \Pi(y)]} \\
& =C^{-1 / 2}(\zeta) \sum_{k}\left(\Phi_{\boldsymbol{k}}(x) \nabla_{\zeta} \Phi_{\boldsymbol{k}}^{*}(y)-\Phi_{\boldsymbol{k}}^{*}(x) \nabla_{\zeta} \Phi_{\boldsymbol{k}}(y)\right) \\
& =(2 \pi)^{-3 / 2} C^{-1 / 2}(\zeta) \frac{3}{2} \zeta^{1 / 2} \sum_{\boldsymbol{k}}\left[\Phi_{-\boldsymbol{k}}(x) A^{*}(-\boldsymbol{k}) e^{i k \cdot y} H_{v}^{(2) *}(k \zeta)\right) \\
& \left.\left.-\Phi_{\boldsymbol{k}}^{*}(x) A(\boldsymbol{k}) e^{i \boldsymbol{k} \cdot \boldsymbol{y}} H_{v}^{(2)}(k \zeta)\right)\right] \\
& +(2 \pi)^{-3 / 2} C^{-1 / 2}(\zeta) \zeta^{3 / 2} \sum_{k}\left[\Phi_{-\boldsymbol{k}}(x) A^{*}(-\boldsymbol{k}) e^{i \boldsymbol{k} \cdot y} \nabla_{\zeta} H_{v}^{(2) *}(k \zeta)\right) \\
& \left.-\Phi_{\boldsymbol{k}}^{*}(x) A(\boldsymbol{k}) e^{i \boldsymbol{k} \cdot \boldsymbol{y}} \nabla_{\zeta}\left(H_{v}^{(2)}(k \zeta)\right)\right] .
\end{aligned}
$$


The first term vanishes; hence,

$$
\begin{aligned}
& {[\phi(x), \Pi(y)]=(2 \pi)^{-3} C^{-1 / 2}(\zeta) \zeta^{3} \sum_{k}|A(k)|^{2}} \\
& \times\left[e^{i \boldsymbol{k} \cdot(\boldsymbol{y}-x)} H_{v}^{(2)}(k \zeta) \nabla_{\zeta} H_{v}^{(2) *}(k \zeta)\right) \\
& \left.-e^{i k \cdot(\boldsymbol{y}-x)} H_{v}^{(2) *}(k \zeta) \nabla_{\zeta}\left(H_{v}^{(2)}(k \zeta)\right)\right] .
\end{aligned}
$$

Using equation (86), we have

$$
[\phi(x), \Pi(y)]_{\mid \Sigma}=i C^{-3 / 2}(\zeta) \delta^{3}(\boldsymbol{y}-\boldsymbol{x}) .
$$

Similarly,

$$
\begin{aligned}
& {[\Pi(x), \Pi(y)] } \\
= & C^{-1}(\zeta) \sum_{k, p}\left[a_{p} \nabla_{\zeta} \Phi_{p}(x)+a_{p}^{\dagger} \nabla_{\zeta} \Phi_{p}^{*}(x), a_{k} \nabla_{\zeta} \Phi_{k}(y)\right. \\
& \left.+a_{k}^{\dagger} \nabla_{\zeta} \Phi_{k}^{*}(y)\right] \\
= & C^{-1}(\zeta) \sum_{p}\left[\dot{g}_{p}(\zeta) \dot{g}_{p}^{*}(\zeta) e^{i p \cdot(x-y)}-\dot{g}_{p}^{*}(\zeta) \dot{g}_{p}(\zeta) e^{-i p \cdot(x-y)}\right]=0 .
\end{aligned}
$$

\subsection{Heisenberg 2nd Quantized Hamiltonian}

As in time-dependent harmonic oscillators, $H \neq H_{ \pm}$[59], we need to discuss Hamiltonian in Heisenberg and Schrödinger picture separately. Denoting $\partial_{\zeta} g_{k}(\zeta)=\dot{g}_{\boldsymbol{k}}(\zeta)$,

$$
\begin{aligned}
H & =\frac{1}{2} \int C^{3 / 2}(\zeta) d^{3} x\left[C^{-1}(\zeta) \sum_{k, p}\left(a_{p} \dot{g}_{p} e^{i p \cdot x}+a_{p}^{\dagger} \dot{g}_{p}^{*} e^{-i p \cdot x}\right)\right. \\
& \times\left(a_{\boldsymbol{k}} \dot{g}_{\boldsymbol{k}} e^{i \boldsymbol{k} \cdot \boldsymbol{x}}+a_{\boldsymbol{k}}^{\dagger} \dot{g}_{\boldsymbol{k}}^{*} e^{-i \boldsymbol{k} \cdot \boldsymbol{x}}\right) \\
& +C^{-1}(\zeta) \sum_{\boldsymbol{k}, \boldsymbol{p}}(i \boldsymbol{p}) \cdot(i \boldsymbol{k})\left(a_{\boldsymbol{p}} g_{\boldsymbol{p}} e^{i \boldsymbol{p} \cdot \boldsymbol{x}}-a_{p}^{\dagger} g_{\boldsymbol{p}}^{*} e^{-i \boldsymbol{p} \cdot \boldsymbol{x}}\right) \\
& \times\left(a_{\boldsymbol{k}} g_{\boldsymbol{k}} e^{i \boldsymbol{k} \cdot \boldsymbol{x}}-a_{\boldsymbol{k}}^{\dagger} g_{\boldsymbol{k}}^{*} e^{-i \boldsymbol{k} \cdot \boldsymbol{x}}\right) \\
& \left.+m_{\mathrm{KG}}^{2} \sum_{\boldsymbol{k}, \boldsymbol{p}}\left(a_{\boldsymbol{p}} g_{\boldsymbol{p}} e^{i \boldsymbol{p} \cdot \boldsymbol{x}}+a_{p}^{\dagger} g_{\boldsymbol{p}}^{*} e^{-i \boldsymbol{p} \cdot \boldsymbol{x}}\right)\left(a_{\boldsymbol{k}} g_{\boldsymbol{k}} e^{i \boldsymbol{k} \cdot \boldsymbol{x}}+a_{\boldsymbol{k}}^{\dagger} g_{\boldsymbol{k}}^{*} e^{-i \boldsymbol{k} \cdot \boldsymbol{x}}\right)\right] .
\end{aligned}
$$

We write

$$
\begin{aligned}
H(\zeta)= & \frac{1}{2} \sum_{k} \varepsilon_{k}(\zeta)\left(a_{k}^{\dagger} a_{k}+a_{k} a_{k}^{\dagger}\right) \\
& +\frac{1}{2} \sum_{k}\left[\Delta_{k}(\zeta) a_{k} a_{-k}+\Delta_{k}^{*}(\zeta) a_{-k}^{\dagger} a_{k}^{\dagger}\right],
\end{aligned}
$$

where

$$
\begin{aligned}
\varepsilon_{k} & =(2 \pi)^{3} C^{1 / 2}(\zeta)\left[\left|\dot{g}_{\boldsymbol{k}}\right|^{2}+\left(\boldsymbol{k}^{2}+C(\zeta) m_{\mathrm{KG}}^{2}\right)\left|g_{\boldsymbol{k}}\right|^{2}\right], \\
\Delta_{\boldsymbol{k}} & =(2 \pi)^{3} C^{1 / 2}(\zeta)\left[\dot{g}_{\boldsymbol{k}}^{2}+\left(\boldsymbol{k}^{2}+C(\zeta) m_{\mathrm{KG}}^{2}\right) g_{k}^{2}\right] .
\end{aligned}
$$

Our expression is slightly different from [52]. The Hamiltonian $H(\zeta)$ can be diagonalized by the Bogliubov transformation:

$$
a_{k}=u_{-k}^{*} b_{k}-v_{k} b_{-k^{\prime}}^{\dagger} \quad a_{k}^{\dagger}=u_{-k} b_{k}^{\dagger}-v_{k}^{*} b_{-k},
$$

where

$$
u_{k}=\sqrt{\frac{\varepsilon_{k}+\omega_{k}(\zeta)}{2 \omega_{k}(\zeta)}}, \quad v_{k}=\frac{\Delta_{k}^{*}}{\varepsilon_{k}+\omega_{k}(\zeta)} u_{k}
$$

and $\omega_{k}(\zeta)=\sqrt{\varepsilon_{k}^{2}-\left|\Delta_{k}\right|^{2}}$. We have

$$
H(\zeta)=\frac{1}{2} \sum_{k} \omega_{k}(\zeta)\left[b_{k}^{\dagger}(\zeta) b_{k}(\zeta)+b_{k}(\zeta) b_{k}^{\dagger}(\zeta)\right]
$$

According to the standard quantum theory of many-body systems [62], $b_{k}^{\dagger}(\zeta)$ generate observed quasi-particles/excitations. From the inverse

$$
\begin{aligned}
& b_{k}(\zeta)=u_{k}(\zeta) a_{k}+v_{k}(\zeta) a_{-k^{\prime}}^{+} \\
& b_{k}^{+}(\zeta)=u_{k}^{*}(\zeta) a_{k}^{+}+v_{k}^{*}(\zeta) a_{-k^{\prime}}
\end{aligned}
$$

we have the commutation relations

$$
\begin{aligned}
& {\left[b_{\boldsymbol{k}}\left(\zeta_{1}\right), b_{p}^{\dagger}\left(\zeta_{2}\right)\right]=\left(u_{\boldsymbol{k}}\left(\zeta_{1}\right) u_{\boldsymbol{k}}^{*}\left(\zeta_{2}\right)-v_{k}\left(\zeta_{1}\right) v_{\boldsymbol{k}}^{*}\left(\zeta_{2}\right)\right) \delta^{3}(\boldsymbol{k}-\boldsymbol{p}),} \\
& {\left[b_{\boldsymbol{k}}\left(\zeta_{1}\right), b_{p}\left(\zeta_{2}\right)\right]=\left(u_{\boldsymbol{k}}\left(\zeta_{1}\right) v_{p}\left(\zeta_{2}\right)-v_{k}\left(\zeta_{1}\right) u_{\boldsymbol{p}}\left(\zeta_{2}\right)\right) \delta^{3}(\boldsymbol{k}+\boldsymbol{p}) .}
\end{aligned}
$$

So, $b_{k}\left(\zeta_{1}\right)$ and $b_{k}\left(\zeta_{2}\right)$ do not commute if $\zeta_{1} \neq \zeta_{2}$. The momentum operator is

$$
P=\sum_{k} k a_{k}^{\dagger} a_{k}=\sum_{k} k b_{k}^{\dagger} b_{k}
$$

$C^{-1 / 2}(\zeta) k$ is the measured momentum. Again using equation (86)

$$
\dot{g}_{k}^{*} g_{k}-\dot{g}_{k} g_{k}^{*}=(2 \pi)^{-3} \ell^{-2} \zeta^{2}(-i)
$$

and the expression

$$
\omega_{k}^{2}=-(2 \pi)^{6} C(\zeta)\left(k^{2}+C(\zeta) m_{\mathrm{KG}}^{2}\right)\left(\dot{g}_{k}^{*} g_{k}-\dot{g}_{k} g_{k}^{*}\right)^{2},
$$

we have

$$
\omega_{\boldsymbol{k}}^{2}(\zeta)=C^{-1}(\zeta)\left(\boldsymbol{k}^{2}+C(\zeta) m_{\mathrm{KG}}^{2}\right)
$$

\subsection{Schrödinger 2nd Quantized Hamiltonian}

In the Schrödinger picture, canonical fields are constant and agree with the fields of the Heisenberg pictures at the chosen time $\zeta=\ell$. The Hamiltonian is then equation (46) with

$$
\begin{aligned}
\phi^{\mathrm{S}}(x) & =\sum_{k}\left(a_{k} \Phi_{k}(\ell, x)+a_{k}^{\dagger} \Phi_{k}^{*}(\ell, x)\right), \\
\pi^{\mathrm{S}}(x) & =\sum_{p}\left(a_{p} \nabla_{\zeta} \Phi_{p}(\ell, x)+a_{p}^{\dagger} \nabla_{\zeta} \Phi_{p}^{*}(\ell, x)\right) .
\end{aligned}
$$

Thus, we have

$$
\begin{aligned}
& H^{S}=\frac{1}{2} C^{3 / 2}(\zeta)(2 \pi)^{3} \sum_{k}\left[C ^ { - 3 } ( \zeta ) \left(\dot{g}_{-k}(\ell) \dot{g}_{k}(\ell) a_{-k} a_{k}\right.\right. \\
& \left.+a_{\boldsymbol{k}} a_{\boldsymbol{k}}^{\dagger} \dot{g}_{\boldsymbol{k}}(\ell) \dot{g}_{\boldsymbol{k}}^{*}(\ell)+a_{\boldsymbol{k}}^{\dagger} a_{\boldsymbol{k}} \dot{g}_{\boldsymbol{k}}^{*}(\ell) \dot{g}_{\boldsymbol{k}}(\ell)+a_{-\boldsymbol{k}}^{+} a_{\boldsymbol{k}}^{\dagger} \dot{g}_{-\boldsymbol{k}}^{*}(\ell) \dot{g}_{\boldsymbol{k}}^{*}(\ell)\right) \\
& +\left(C^{-1}(\zeta) k^{2}+m_{\mathrm{KG}}^{2}\right)\left(a_{-k} a_{k} g_{-k}(\ell) g_{k}(\ell)+a_{k} a_{k}^{\dagger} g_{k}(\ell) g_{k}^{*}(\ell)\right. \\
& \left.\left.+a_{k}^{\dagger} a_{k} g_{k}^{*}(\ell) g_{k}(\ell)+a_{-k}^{\dagger} a_{k}^{\dagger} g_{-k}^{*}(\ell) g_{k}^{*}(\ell)\right)\right]
\end{aligned}
$$

As in the Heisenberg picture, we write

$$
\begin{aligned}
H^{\mathrm{S}}(\zeta) & =\frac{1}{2} \sum_{k} \varepsilon_{k}^{\mathrm{S}}(\zeta)\left(a_{k}^{\dagger} a_{k}+a_{k} a_{k}^{\dagger}\right) \\
& +\frac{1}{2} \sum_{k}\left[\Delta_{k}^{\mathrm{S}}(\zeta) a_{k} a_{-k}+\Delta_{k}^{\mathrm{S} *}(\zeta) a_{-k}^{\dagger} a_{k}^{\dagger}\right]
\end{aligned}
$$


where

$$
\begin{aligned}
& \varepsilon_{k}^{\mathrm{S}}=(2 \pi)^{3} C^{1 / 2}(\zeta)\left[\frac{\left|\dot{g}_{k}(\ell)\right|^{2}}{C^{2}(\zeta)}+\left(\boldsymbol{k}^{2}+C(\zeta) m_{\mathrm{KG}}^{2}\right)\left|g_{\boldsymbol{k}}(\ell)\right|^{2}\right], \\
& \Delta_{k}^{\mathrm{S}}=(2 \pi)^{3} C^{1 / 2}(\zeta)\left[C^{-2}(\zeta) \dot{g}_{k}^{2}(\ell)+\left(\boldsymbol{k}^{2}+C(\zeta) m_{\mathrm{KG}}^{2}\right) g_{\boldsymbol{k}}^{2}(\ell)\right] .
\end{aligned}
$$

By the same token, one can calculate

$$
\begin{aligned}
\omega_{\boldsymbol{k}}^{\mathrm{S} 2}(\zeta)= & -(2 \pi)^{6} C^{-1}(\zeta)\left(\boldsymbol{k}^{2}+C(\zeta) m_{\mathrm{KG}}^{2}\right) \\
& \times\left(\dot{g}_{\boldsymbol{k}}(\ell)^{*} g_{\boldsymbol{k}}(\ell)-\dot{g}_{\boldsymbol{k}}(\ell) g_{\boldsymbol{k}}^{*}(\ell)\right)^{2} .
\end{aligned}
$$

At $\zeta=\ell, \dot{g}_{\boldsymbol{k}}^{*}(\ell) g_{\boldsymbol{k}}(\ell)-\dot{g}_{\boldsymbol{k}}(\ell) g_{\boldsymbol{k}}^{*}(\ell)=(2 \pi)^{-3}(-i)$. Hence, $\omega_{k}^{\mathrm{S} 2}(\zeta)=\omega_{k}^{2}$. The Bogliubov transformation is

$$
\begin{aligned}
b_{k}^{\mathrm{S}}(\zeta) & =u_{k}^{\mathrm{S}}(\zeta) a_{k}+v_{k}^{\mathrm{S}}(\zeta) a_{-k^{\prime}}^{+} \\
b_{k}^{\mathrm{S}}(\zeta) & =u_{k}^{\mathrm{S} *}(\zeta) a_{k}^{\dagger}+v_{k}^{\mathrm{S} *}(\zeta) a_{-k^{\prime}}
\end{aligned}
$$

with

$$
u_{k}^{S}=\sqrt{\frac{\varepsilon_{k}^{S}+\omega_{k}(\zeta)}{2 \omega_{k}(\zeta)}}, \quad v_{k}^{S}=\frac{\Delta_{k}^{S *}}{\varepsilon_{k}^{S}+\omega_{k}(\zeta)} u_{k}^{S} .
$$

The Schrödinger picture Hamiltonian is

$$
H^{\mathrm{S}}(\zeta)=\frac{1}{2} \sum_{k} \omega_{k}(\zeta)\left(b_{k}^{\mathrm{S}}(\zeta) b_{k}^{\mathrm{S}}(\zeta)+b_{k}^{\mathrm{S}}(\zeta) b_{k}^{\mathrm{S} \dagger}(\zeta)\right) \neq H(\zeta)
$$

The relation between quasi-creation/annihilation operators $b_{k}(\zeta)$ and $b_{k}^{\mathrm{S}}(\zeta)$ is

$$
\begin{aligned}
b_{k}^{\mathrm{S}}(\zeta)= & {\left[u^{\mathrm{S}}(\zeta) u_{k}^{*}(\zeta)-v_{k}^{\mathrm{S}}(\zeta) v_{k}^{*}(\zeta)\right] b_{k}(\zeta) } \\
& +\left[v^{\mathrm{S}}(\zeta) u_{k}(\zeta)-u_{k}^{\mathrm{S}}(\zeta) v_{k}(\zeta)\right] b_{-k}^{\dagger}(\zeta), \\
b_{k}^{\mathrm{S}}(\zeta)= & {\left[u^{\mathrm{S} *}(\zeta) u_{k}(\zeta)-v_{k}^{\mathrm{S} *}(\zeta) v_{k}(\zeta)\right] b_{k}^{\dagger}(\zeta) } \\
& +\left[v^{\mathrm{S} *}(\zeta) u_{k}^{*}(\zeta)-u_{k}^{\mathrm{S} *}(\zeta) v_{k}^{*}(\zeta)\right] b_{-k}(\zeta),
\end{aligned}
$$

and the inverse is

$$
\begin{aligned}
b_{k}(\zeta)= & {\left[u(\zeta) u_{k}^{\mathrm{S} *}(\zeta)-v_{k}(\zeta) v_{k}^{\mathrm{S} *}(\zeta)\right] b_{k}^{\mathrm{S}}(\zeta) } \\
& +\left[v(\zeta) u_{k}^{\mathrm{S}}(\zeta)-u_{k}(\zeta) v_{k}^{\mathrm{S}}(\zeta)\right] b_{-k}^{\mathrm{S}+}(\zeta), \\
b_{k}^{\dagger}(\zeta)= & {\left[u^{*}(\zeta) u_{k}^{\mathrm{S}}(\zeta)-v_{k}^{*}(\zeta) v_{k}^{\mathrm{S}}(\zeta)\right] b_{k}^{\mathrm{S}}(\zeta) } \\
& +\left[v^{*}(\zeta) u_{k}^{\mathrm{S}^{*}}(\zeta)-u_{k}^{*}(\zeta) v_{k}^{\mathrm{S} *}(\zeta)\right] b_{-k}^{\mathrm{S}}(\zeta) .
\end{aligned}
$$

These relations are important to perturbative calculations, as will be discussed later on. The measured energy-momentum is [11]

$$
p_{a}(\zeta)=\left(\omega_{k}, C^{-1 / 2}(\zeta) k\right)
$$

So, we have an-shell relation of the measured 4-momentum $p_{a}(\zeta) p^{a}(\zeta)=m_{\mathrm{KG}}^{2}$ as in the Minkowski spacetime. We have accordingly the redshift relation

$$
\frac{\omega_{\boldsymbol{k}}\left(\zeta_{1}\right)}{\omega_{\boldsymbol{k}}\left(\zeta_{2}\right)}=\sqrt{\frac{C^{-1}\left(\zeta_{1}\right)\left(\boldsymbol{k}^{2}+C\left(\zeta_{1}\right) m_{\mathrm{KG}}^{2}\right)}{C^{-1}\left(\zeta_{2}\right)\left(\boldsymbol{k}^{2}+C\left(\zeta_{2}\right) m_{\mathrm{KG}}^{2}\right)}} .
$$

This relation seems new to the best of our knowledge. Since many existing discussions lack both unique and invariant definitions of observable quantum particle states, concepts such as energy are not well defined. The impact of a curved spacetime on the measured energy of particles was discussed via phase analysis [10], and the behavior of $\omega_{k}(\zeta)$ here agrees exactly with the geometric-optics limit [11]. This redshift relation agrees with the conventional relation $\omega_{B} / \omega_{A}=$ $\sqrt{g_{00}\left(x_{A}\right) / g_{00}\left(x_{B}\right)}$ in the zero-mass limit.

Similar to the vacuum state of the Fock space of operators $a_{k}$ and $a_{k}^{+}$

$$
|0\rangle=\prod_{k}\left|0_{k}\right\rangle, a_{k}\left|0_{k}\right\rangle=0,
$$

we define $|\mathbf{O} ; \zeta\rangle^{\mathrm{S}}$ s.t.

$$
b_{k}^{S}(\zeta)|\mathbf{O} ; \zeta\rangle^{S}=0, \quad \forall \quad k,
$$

i.e.,

$$
|\mathbf{O} ; \zeta\rangle^{S}=\prod_{k}\left|\mathbf{O}_{k} ; \zeta\right\rangle_{k^{\prime}}^{S}
$$

where

$$
b_{k}^{S}\left|\mathbf{O}_{k} ; \zeta\right\rangle_{k}^{S}=0
$$

Since

$$
\langle 0|H(\zeta)| 0\rangle=\sum_{k} \varepsilon_{k}>{ }^{\mathrm{S}}\langle\mathbf{O} ; \zeta|H(\zeta)| \mathbf{O} ; \zeta\rangle^{\mathrm{S}}=\sum_{k} \omega_{k}(\zeta)
$$

$|0\rangle$ is not the ground state. Here, we have a system of timedependent oscillators, as the cases discussed in [54]-[60]. The vacuum states are time-dependent. The vacuum state at one time will evolve into a nonvacuum state at a later time. Thus, particles can be generated.

In the remaining part of this section and the next, states and generation/annihilation operators $b_{k}^{\dagger}(\zeta)$ and $b_{k}(\zeta)$ can be in either Schrödinger picture or Heisenberg pictures since the math structures are essentially the same. Denote that

$$
\left|\left\{n_{\boldsymbol{k}}\right\} ; \zeta\right\rangle=\prod_{i}\left\{\frac{1}{\sqrt{n_{\boldsymbol{k}_{i}} !}}\left[b_{\boldsymbol{k}_{i}}^{\dagger}(\zeta)\right]^{n_{\boldsymbol{k}_{i}}}\left|\mathbf{O}_{k_{i}} ; \zeta\right\rangle_{\boldsymbol{k}_{i}}\right\}
$$

and $\left|1_{k} ; \zeta\right\rangle=|k ; \zeta\rangle$. Using the results in [53], we have

$$
|\mathbf{O} ; \zeta\rangle=\hat{A}(\zeta)|0\rangle=\otimes_{k}\left|\mathbf{O}_{k} ; \zeta\right\rangle_{k}
$$

where

$$
\hat{A}(\zeta)=\mathcal{A}(\zeta) e^{-\frac{1}{2} \sum_{k} \frac{v_{k}(\zeta)}{u_{k}(\zeta)} a_{-k}^{\dagger} a_{k}^{+}}
$$

with

$$
\mathcal{A}(\zeta)=\prod_{k}{ }^{\prime} \mathcal{A}_{k}(\zeta)
$$

where $\prod_{k}{ }^{\prime}$ means that only one of the two factors corresponding to $k,-k$ appears in the product and $\mathcal{A}_{k}(\zeta)=1 / u_{k}(\zeta)$. Denoting

$$
\gamma_{p}(\zeta)=\frac{v_{p}(\zeta)}{u_{p}(\zeta)}=\frac{\theta_{k}^{*}(\zeta)}{\left|\theta_{k}^{*}(\zeta)\right|} \operatorname{th}\left|\theta_{k}(\zeta)\right|
$$

then

$$
\hat{A}(\zeta)=\mathcal{A}(\zeta) e^{-\frac{1}{2} \sum_{k} \gamma_{k}(\zeta) a_{-k}^{+} a_{k}^{+}}=\prod_{k}{ }^{\prime} \hat{A}_{k}(\zeta)
$$


with

$$
\hat{A}_{k}(\zeta)=\mathcal{A}_{k}(\zeta) e^{-\gamma_{k}(\zeta) a_{-k}^{+} a_{k}^{+}}=\hat{A}_{-k}(\zeta)
$$

For a particlular $k$,

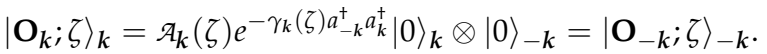

We can calculate $\left\langle\mathbf{O} ; \zeta_{1} \mid \mathbf{O} ; \zeta_{2}\right\rangle$. First, we have

$$
{ }_{k}\left\langle\mathbf{O}_{k} ; \zeta_{1} \mid \mathbf{O}_{k} ; \zeta_{2}\right\rangle_{k}=\frac{1}{u_{k}\left(\zeta_{1}\right) u_{k}\left(\zeta_{2}\right)-v_{k}^{*}\left(\zeta_{1}\right) v_{k}\left(\zeta_{2}\right)} .
$$

Then,

$$
\left\langle\mathbf{O} ; \zeta_{1} \mid \mathbf{O} ; \zeta_{2}\right\rangle=\prod_{k} \frac{1}{\sqrt{u_{k}\left(\zeta_{1}\right) u_{k}\left(\zeta_{2}\right)-v_{k}^{*}\left(\zeta_{1}\right) v_{k}\left(\zeta_{2}\right)}} .
$$

Using the following expectations,

$$
\begin{aligned}
& \left\langle\mathbf{O} ; \zeta^{\prime \prime}\left|a_{k} a_{p}\right| \mathbf{O} ; \zeta^{\prime}\right\rangle \\
= & \left\{\begin{array}{cl}
0, & p \neq \pm \boldsymbol{k}, \boldsymbol{p}=\boldsymbol{k}, \\
\left\langle\mathbf{O} ; \zeta^{\prime \prime} \mid \mathbf{O} ; \zeta^{\prime}\right\rangle \frac{-1}{1-\gamma_{k}^{*}\left(\zeta^{\prime \prime}\right) \gamma_{k}\left(\zeta^{\prime}\right)}, & \boldsymbol{p}=-\boldsymbol{k},
\end{array}\right. \\
& \left\langle\mathbf{O} ; \zeta^{\prime \prime}\left|a_{\boldsymbol{k}} a_{p}^{\dagger}\right| \mathbf{O} ; \zeta^{\prime}\right\rangle \\
= & \left\{\begin{array}{cl}
0, & p \neq \pm \boldsymbol{k}, \boldsymbol{p}=-\boldsymbol{k}, \\
\left\langle\mathbf{O} ; \zeta^{\prime \prime} \mid \mathbf{O} ; \zeta^{\prime}\right\rangle \frac{1}{1-\gamma_{k}^{*}\left(\zeta^{\prime \prime}\right) \gamma_{k}\left(\zeta^{\prime}\right)}, & \boldsymbol{p}=\boldsymbol{k},
\end{array}\right.
\end{aligned}
$$

one can calculate

$$
\begin{aligned}
& \left\langle\mathbf{O} ; \zeta^{\prime \prime}\left|b_{k}\left(\zeta^{\prime \prime}\right) b_{k}^{\dagger}\left(\zeta^{\prime}\right)\right| \mathbf{O} ; \zeta^{\prime}\right\rangle \\
= & \left\langle\mathbf{O} ; \zeta^{\prime \prime} \mid \mathbf{O} ; \zeta^{\prime}\right\rangle \times{ }_{k}\left\langle\mathbf{O}_{k} ; \zeta^{\prime \prime} \mid \mathbf{O}_{k} ; \zeta^{\prime}\right\rangle_{k} .
\end{aligned}
$$

Hence,

$$
\left.{ }_{k}\left\langle\mathbf{O}_{k} ; \zeta^{\prime \prime}\left|b_{k}\left(\zeta^{\prime \prime}\right) b_{k}^{\dagger}\left(\zeta^{\prime}\right)\right| \mathbf{O}_{k} ; \zeta^{\prime}\right\rangle_{k}={ }_{k}\left\langle\mathbf{O}_{k} ; \zeta^{\prime \prime} \mid \mathbf{O}_{k} ; \zeta^{\prime}\right\rangle_{k}\right]^{2}
$$

Similarly,

$$
\begin{aligned}
& { }_{k}\left\langle\mathbf{O}_{k} ; \zeta^{\prime \prime}\left|b_{k}\left(\zeta^{\prime \prime}\right) b_{-k}\left(\zeta^{\prime \prime}\right)\right| \mathbf{O}_{k} ; \zeta^{\prime}\right\rangle_{k} \\
& =\frac{{ }_{k}\left\langle\mathbf{O}_{k} ; \zeta^{\prime \prime} \mid \mathbf{O}_{k} ; \zeta^{\prime}\right\rangle_{k}}{1-\gamma_{k}^{*}\left(\zeta^{\prime \prime}\right) \gamma_{k}\left(\zeta^{\prime}\right)}\left[\gamma_{k}\left(\zeta^{\prime \prime}\right)-\gamma_{k}\left(\zeta^{\prime}\right)\right] \text {. }
\end{aligned}
$$

\section{GENERATING FUNCTION OF VACUUM EXPECTATIONS}

We define the generating function

$$
\begin{aligned}
& z\left[\lambda, \lambda^{*} ; \zeta, \zeta^{\prime}\right] \\
:= & \prod_{\boldsymbol{k}}{ }_{k}\left\langle\mathbf{O}_{\boldsymbol{k}} ; \zeta\right| e^{\int d \eta \lambda_{k}(\eta) b_{k}(\eta)} e^{\int d \eta \lambda_{-k}(\eta) b_{-k}(\eta)} \\
& \times e^{\int d \eta^{\prime}\left(\lambda_{k}^{*}\left(\eta^{\prime}\right) b_{k}^{+}\left(\eta^{\prime}\right)\right.} e^{\int d \eta^{\prime} \lambda_{-k}^{*}\left(\eta^{\prime}\right) b_{-k}^{+}\left(\eta^{\prime}\right)}\left|\mathbf{O}_{\boldsymbol{k}} ; \zeta^{\prime}\right\rangle_{\boldsymbol{k}} .
\end{aligned}
$$

$\lambda_{k}(\eta)$ and $\lambda_{k}^{*}(\eta)$ are considered as external fields and are independent of each other, instead of being mutually complex conjugate. For a particular $k$, we define

$$
\begin{aligned}
& z_{k}\left[\lambda, \lambda^{*} ; \zeta, \zeta^{\prime}\right] \\
:= & \left\langle\mathbf{O}_{k} ; \zeta\right| e^{\int d \eta \lambda_{k}(\eta) b_{k}(\eta)} e^{\int d \eta \lambda_{-k}(\eta) b_{-k}(\eta)} \\
& \times e^{\int d \eta^{\prime}\left(\lambda_{k}^{*}\left(\eta^{\prime}\right) b_{k}^{\dagger}\left(\eta^{\prime}\right)\right.} e^{\int d \eta^{\prime} \lambda_{-k}^{*}\left(\eta^{\prime}\right) b_{-k}^{+}\left(\eta^{\prime}\right)}\left|\mathbf{O}_{\boldsymbol{k}} ; \zeta^{\prime}\right\rangle_{\boldsymbol{k}} \\
= & { }_{\boldsymbol{k}}\left\langle\mathbf{O}_{\boldsymbol{k}} ; \zeta\right| e^{\int d \eta \lambda_{k}(\eta)\left[u_{k}(\eta) a_{k}+v_{k}(\eta) a_{-k}^{+}\right]} \\
& \times e^{\int d \eta \lambda_{-k}(\eta)\left[u_{-k}(\eta) a_{-k}+v_{-k}(\eta) a_{k}^{+}\right]} \\
& \times e^{\int d \eta^{\prime} \lambda_{k}^{*}\left(\eta^{\prime}\right)\left[u_{k}^{*}\left(\eta^{\prime}\right) a_{k}^{+}+v_{k}^{*}\left(\eta^{\prime}\right) a_{-k}\right]} \\
& \times e^{\int d \eta^{\prime} \lambda_{-k}^{*}\left(\eta^{\prime}\right)\left[u_{-k}^{*}\left(\eta^{\prime}\right) a_{-k}^{+}+v_{-k}^{*}\left(\eta^{\prime}\right) a_{k}\right]}\left|\mathbf{O}_{k} ; \zeta^{\prime}\right\rangle_{k} .
\end{aligned}
$$

Denoting

$$
\alpha_{k}[\lambda]=\int d \eta \lambda_{k}(\eta) u_{k}(\eta), \quad \beta_{k}[\lambda]=\int d \eta \lambda_{k}(\eta) v_{k}(\eta),
$$

$$
\begin{aligned}
z_{k}\left[\lambda, \lambda^{*} ; \zeta, \zeta^{\prime}\right] & ={ }_{k}\left\langle\mathbf{O}_{k} ; \zeta\right| e^{\alpha_{k} a_{k}} \cdot e^{\beta_{k} a_{-k}^{+}} \cdot e^{\alpha_{-k} a_{-k}} \cdot e^{\beta_{-k} a_{k}^{+}} \\
& \times e^{\alpha_{k}^{*} a_{k}^{+}} \cdot e^{\beta_{k}^{*} a_{-k}} \cdot e^{\alpha_{-k}^{*} a_{-k}^{+}} \cdot e^{\beta_{-k}^{*} a_{k}}\left|\mathbf{O}_{k} ; \zeta^{\prime}\right\rangle_{k} .
\end{aligned}
$$

Using coherent states $\left|z_{k}, z_{-k}\right\rangle=e^{z_{k} a_{k}^{\dagger}+z_{-k} a_{-k}^{\dagger}}|0\rangle$, one can calculate

$$
\begin{aligned}
& z_{k}\left[\lambda, \lambda^{*} ; \zeta, \zeta^{\prime}\right] \\
= & \frac{\mathcal{A}_{k}\left(\zeta^{\prime}\right) \mathcal{A}_{k}^{*}(\zeta)}{1-\gamma_{k}^{*}(\zeta) \gamma_{k}\left(\zeta^{\prime}\right)} \\
& \times \exp \left[-\beta_{-k}^{*}\left(\alpha_{k}^{*}+\beta_{-k}\right)-\beta_{k}\left(\alpha_{-k}+\beta_{k}^{*}\right)\right. \\
& +\frac{1}{1-\gamma_{k}^{*}(\zeta) \gamma_{k}\left(\zeta^{\prime}\right)}\left(A_{k}^{*} A_{k}+A_{-k}^{*} A_{-k}\right. \\
& \left.\left.-A_{-k}^{*} \gamma_{k}\left(\zeta^{\prime}\right) A_{k}^{*}-A_{k} \gamma_{k}^{*}(\zeta) A_{-k}\right)\right],
\end{aligned}
$$

with which the following amplitudes can be evaluated:

$$
\begin{aligned}
{ }_{k}\left\langle 1_{k} ; \zeta \mid 1_{k} ; \zeta^{\prime}\right\rangle_{k} & =\frac{\delta}{\delta \lambda_{k}(\zeta)} \frac{\delta}{\delta \lambda_{k}^{*}\left(\zeta^{\prime}\right)} z_{k}\left[\lambda, \lambda^{*} ; \zeta, \zeta^{\prime}\right] \mid \lambda=\lambda^{*}=0 \\
& =\frac{1}{\left[u_{k}(\zeta) u_{k}\left(\zeta^{\prime}\right)\left(1-\gamma_{k}^{*}(\zeta) \gamma_{k}\left(\zeta^{\prime}\right)\right]^{2}\right.}
\end{aligned}
$$

and

$$
\begin{aligned}
& { }_{k}\left\langle 1_{k} 1_{-k} ; \zeta \mid \mathbf{O} ; \zeta^{\prime}\right\rangle_{k} \\
= & \frac{\delta}{\delta \lambda_{k}(\zeta)} \frac{\delta}{\delta \lambda_{-k}(\zeta)} z_{k}\left[\lambda, \lambda^{*} ; \zeta, \zeta^{\prime}\right]_{\mid \lambda=\lambda^{*}=0} \\
= & \frac{\mathcal{A}_{k}\left(\zeta^{\prime}\right) \mathcal{A}_{k}^{*}(\zeta)}{\left[1-\gamma_{k}^{*}(\zeta) \gamma_{k}\left(\zeta^{\prime}\right]^{2}\right.}\left(\gamma(\zeta)-\gamma\left(\zeta^{\prime}\right)\right) .
\end{aligned}
$$

\section{TRANSITION OF STATES OF FREE KG FIELD}

As in conventional quantum field theories [63], suppose that at time $\zeta_{1}$, the system is in a vacuum state, and the state at $\zeta_{2}$ has some computable probability to contain multiple particles. In the Schrödinger picture, the state

$$
\left|\Psi_{0}\left(\zeta_{2} ; \zeta_{1}\right)\right\rangle^{\mathrm{S}}=\hat{T} e^{-i \int_{\zeta_{1}}^{\zeta_{2}} H^{\mathrm{S}}[\phi(x), \pi(x) ; \eta] e_{\zeta}^{0}(\eta) d \eta}\left|\mathbf{O} ; \zeta_{1}\right\rangle^{\mathrm{S}}
$$


is a formal solution to

$$
i \hat{\nabla}_{0}\left|\Psi_{0}\left(\zeta ; \zeta_{1}\right)\right\rangle^{S}=H^{S}[\phi(x), \pi(x) ; \zeta]\left|\Psi_{0}\left(\zeta ; \zeta_{1}\right)\right\rangle^{S}
$$

with initial condition $\left|\Psi_{0}\left(\zeta_{1} ; \zeta_{1}\right)\right\rangle^{S}=\left|\mathbf{O} ; \zeta_{1}\right\rangle^{S}$. The transition amplitude from state $\left|\left\{n_{k}\right\} ; \zeta_{1}\right\rangle^{\mathrm{S}}$ to state $\left|\left\{m_{\boldsymbol{k}}\right\} ; \zeta_{2}\right\rangle^{\mathrm{S}}$ is given by

$$
\begin{aligned}
& \mathscr{T}\left(\left|\left\{n_{\boldsymbol{k}}\right\} ; \zeta_{1}\right\rangle^{\mathrm{S}} \rightarrow\left|\left\{m_{\boldsymbol{k}}\right\} ; \zeta_{2}\right\rangle^{\mathrm{S}}\right) \\
= & { }^{\mathrm{S}}\left\langle\left\{m_{\boldsymbol{k}}\right\} ; \zeta_{2}\left|\hat{T} e^{-i \int_{\zeta_{1}}^{\zeta_{2}} H^{\mathrm{S}}(\eta) e_{\zeta}^{0}(\eta) d \eta}\right|\left\{n_{\boldsymbol{k}}\right\} ; \zeta_{1}\right\rangle^{\mathrm{S}} .
\end{aligned}
$$

For a particular $k$,

$$
\begin{aligned}
& \mathscr{T}\left(\left|m_{\boldsymbol{k}}, m_{-\boldsymbol{k}} ; \zeta_{1}\right\rangle_{\boldsymbol{k}}^{\mathrm{S}} \rightarrow\left|n_{\boldsymbol{k}}, n_{-\boldsymbol{k}} ; \zeta_{2}\right\rangle_{\boldsymbol{k}}^{\mathrm{S}}\right) \\
& ={ }_{\boldsymbol{k}}^{\mathrm{S}}\left\langle n_{\boldsymbol{k}}, n_{-\boldsymbol{k}} ; \zeta_{2}\right| \hat{T} e^{-i \int_{\zeta_{1}}^{\zeta_{2}} \omega_{\boldsymbol{k}}(\eta)\left[b_{k}^{S+}(\eta) b_{k}^{\mathrm{S}}(\eta)+b_{-k}^{\mathrm{St}}(\eta) b_{-\boldsymbol{k}}^{\mathrm{S}}(\eta)+1\right] e_{\zeta}^{0}(\eta) d \eta} \\
& \times\left|m_{\boldsymbol{k}}, m_{-\boldsymbol{k}} ; \zeta_{1}\right\rangle_{\boldsymbol{k}}^{\mathrm{S}} .
\end{aligned}
$$

Slicing the time as $\eta_{0}=\zeta_{1}, \eta_{j}=\eta_{j-1}-\Delta, \Delta=\frac{\zeta_{1}-\zeta_{2}}{N}$, and $\eta_{N}=\zeta_{2}$,

$$
\begin{aligned}
& \mathscr{T}\left(\left|m_{k}, m_{-k} ; \zeta_{1}\right\rangle_{k}^{S} \rightarrow\left|n_{k}, n_{-k} ; \zeta_{2}\right\rangle_{k}^{S}\right) \\
& =\lim _{N \rightarrow \infty}{ }_{k}\left\langle n_{k}, n_{-k} ; \zeta_{2}\right| \\
& \times e^{-i \omega_{k}\left(\eta_{N-1}\right)\left[b_{k}^{S t}\left(\eta_{N-1}\right) b_{k}^{S}\left(\eta_{N-1}\right)+b_{-k}^{S+}\left(\eta_{N_{1}}\right) b_{-k}^{\mathrm{S}}\left(\eta_{N-1}\right)+1\right] e_{\zeta}^{0}\left(\eta_{N-1}\right) \Delta} \\
& \times e^{-i \omega_{k}\left(\eta_{N-2}\right)\left[b_{k}^{S \dagger}\left(\eta_{N-2}\right) b_{k}^{S}\left(\eta_{N-2}\right)+b_{-k}^{S \dagger}\left(\eta_{N_{2}}\right) b_{-k}^{\mathrm{S}}\left(\eta_{N-2}\right)+1\right] e_{\zeta}^{0}\left(\eta_{N-2}\right) \Delta} \\
& \times \cdots e^{i \omega_{k}\left(\eta_{1}\right)\left[b_{k}^{\mathrm{St}}\left(\eta_{1}\right) b_{k}^{\mathrm{S}}\left(\eta_{1}\right)+b_{-k}^{\mathrm{St}}\left(\eta_{1}\right) b_{-k}^{\mathrm{S}}\left(\eta_{1}\right)+1\right] e_{\zeta}^{0}\left(\eta_{1}\right) \Delta} \\
& \times e^{-i \omega_{k}\left(\eta_{0}\right)\left[b_{k}^{\mathrm{St}}\left(\eta_{0}\right) b_{k}^{\mathrm{S}}\left(\eta_{0}\right)+b_{-\boldsymbol{k}}^{\mathrm{St}}\left(\eta_{0}\right) b_{-\boldsymbol{k}}^{\mathrm{S}}\left(\eta_{0}\right)+1\right] e_{\zeta}^{0}\left(\eta_{0}\right) \Delta}\left|m_{\boldsymbol{k}}, m_{-\boldsymbol{k}} ; \zeta_{1}\right\rangle_{\boldsymbol{k}}^{\mathrm{S}} \text {. }
\end{aligned}
$$

Using coherent states $j=0,1, \cdots, N$,

$$
\begin{aligned}
& \left|z_{j k}, z_{j-k}\right\rangle=e^{z_{j k} b_{k}^{S \dagger}\left(\eta_{j}\right)+z_{j-k} b_{-k}^{S \dagger}\left(\eta_{j}\right)}\left|\mathbf{O}_{k} ; \eta_{j}\right\rangle_{\boldsymbol{k}}, \\
& \mathscr{T}\left(\left|m_{k}, m_{-k} ; \zeta_{1}\right\rangle_{k}^{S} \rightarrow\left|n_{k}, n_{-k} ; \zeta_{2}\right\rangle_{k}^{S}\right) \\
& =\lim _{N \rightarrow \infty} e^{-i \int_{\zeta_{1}}^{\zeta_{2}} \omega_{k}(\eta) e_{\zeta}^{0}(\eta) d \eta} \int\left[\prod_{j=0}^{N} \frac{d z_{j k} d z_{j k}^{*}}{2 \pi i}\right] \\
& \times \frac{z_{N k}^{n_{k}}}{\sqrt{n_{k} !}} \frac{z_{0 k}^{* m_{k}}}{\sqrt{m_{k} !}} \exp \left[-z_{0 k}^{*} z_{0 k}\right] \\
& \times \prod_{j=1}^{N} \exp \left[-z_{j k}^{*} z_{j k}+z_{j k}^{*} z_{j-1, k}-i \omega_{k}\left(\eta_{j-1}\right) z_{j k}^{*} z_{j-1 k} e_{\zeta}^{0}\left(\eta_{j-1}\right) \Delta\right] \\
& \times \int\left[\prod_{j=0}^{N} \frac{d z_{j-k} d z_{j-k}^{*}}{2 \pi i}\right] \frac{z_{N-k}^{n_{-k}}}{\sqrt{n_{-k} !}} \frac{z_{0-k}^{* m_{-k}}}{\sqrt{m_{-k} !}} \exp \left[-z_{0-k}^{*} z_{0-k}\right] \\
& \times \prod_{j=1}^{N} \exp \left[-z_{j-k}^{*} z_{j-k}+z_{j-k}^{*} z_{j-1,-k}\right. \\
& \left.-i \omega_{k}\left(\eta_{j-1}\right) z_{j-k}^{*} z_{j-1,-k} e_{\zeta}^{0}\left(\eta_{j-1}\right) \Delta\right],
\end{aligned}
$$

which can be written as a path integral by shorthand. It is easy to see that starting from a vacuum state at $\zeta_{1}$, the state will evolve into a mixed state at later time $\zeta_{2}$, which is not unusual for systems in external fields in the Minkowski quantum field theories [63] and time-dependent harmonic oscillators [54]-[60].

\section{PERTURBATION}

The full Lagrangian of $\lambda^{4}$-theory is

$$
\mathscr{L}=\frac{1}{2}\left(\hat{\nabla}^{a} \phi \hat{\nabla}_{a} \phi-m_{\mathrm{KG}}^{2} \phi^{2}-\frac{\lambda}{4 !} \phi^{4}\right),
$$

and the full Hamiltonian is

$$
\begin{aligned}
\mathscr{H} & =\hat{\nabla}_{0} \phi \cdot \Pi-\mathscr{L}=\mathscr{H}_{0}+\mathscr{H}_{\mathrm{I}} \\
H[\phi, \Pi ; \zeta] & =H_{0}[\phi, \Pi ; \zeta]+H_{\mathrm{I}}[\phi, \Pi ; \zeta],
\end{aligned}
$$

where

$$
\begin{aligned}
\mathscr{H}_{0} & =\frac{1}{2}\left(\Pi^{2}-\hat{\nabla}^{a^{\prime}} \phi \hat{\nabla}_{a^{\prime}} \phi+m_{\mathrm{KG}}^{2} \phi^{2}\right) \\
\mathscr{H}_{\mathrm{I}} & =\frac{1}{2} \frac{\lambda}{4 !} \phi^{4} .
\end{aligned}
$$

The Schrödinger state follows

$$
i \hat{\nabla}_{0}|\Psi(\zeta)\rangle^{\mathrm{S}}=H\left[\phi^{\mathrm{S}}(\boldsymbol{x}), \pi^{\mathrm{S}}(\boldsymbol{x}) ; \zeta\right]|\Psi(\zeta)\rangle^{\mathrm{S}} .
$$

Defining the Dirac picture state

$$
|\Psi(\zeta)\rangle^{\mathrm{D}}:=\hat{T}^{-1} e^{i \int_{\ell}^{\zeta} H_{0}^{\mathrm{S}}(\eta) e_{\zeta}^{0}(\eta) d \eta}|\Psi(\zeta)\rangle^{\mathrm{S}},
$$

hence the two pictures coincide at time $\zeta=\ell,|\Psi(\ell)\rangle^{\mathrm{S}}=$ $|\Psi(\ell)\rangle^{\mathrm{D}}$ and we thus have the equation of motion

$$
i \hat{\nabla}_{0}|\Psi(\zeta)\rangle^{\mathrm{D}}=H_{\mathrm{I}}^{\mathrm{D}}(\zeta)|\Psi(\zeta)\rangle^{\mathrm{D}}
$$

where

$$
H_{\mathrm{I}}^{\mathrm{D}}(\zeta)=U_{0}^{\mathrm{S}-1}(\zeta, \ell) H_{\mathrm{I}}^{\mathrm{S}}(\zeta) U_{0}^{\mathrm{S}}(\zeta, \ell)
$$

with

$$
U_{0}^{S}\left(\zeta^{\prime \prime}, \zeta^{\prime}\right)=\hat{T} e^{-i \int_{\zeta^{\prime}}^{\zeta^{\prime \prime}} H_{0}^{S}(\eta) e_{\zeta}^{0}(\eta) d \eta}
$$

The Dirac picture field and operators are defined as

$$
\begin{aligned}
\phi^{\mathrm{D}}(\zeta, x) & :=U_{0}^{S-1}(\zeta, \ell) \phi^{\mathrm{S}}(\boldsymbol{x}) U_{0}^{\mathrm{S}}(\zeta, \ell), \\
H_{0}^{\mathrm{D}}(\zeta) & :=U_{0}^{\mathrm{S}-1}(\zeta, \ell) H_{0}^{\mathrm{S}}(\zeta) U_{0}^{\mathrm{S}}(\zeta, \ell),
\end{aligned}
$$

from which it follows that

$$
\begin{aligned}
& i e_{0}^{\zeta}(\zeta) \frac{\partial}{\partial \zeta} \phi^{\mathrm{D}}(\zeta, x)=\left[\phi^{\mathrm{D}}(\zeta, x), H_{0}^{\mathrm{D}}\right] \\
& i e_{0}^{\zeta}(\zeta) \frac{\partial}{\partial \zeta} \pi^{\mathrm{D}}(\zeta, x)=\left[\pi^{\mathrm{D}}(\zeta, x), H_{0}^{\mathrm{D}}\right] .
\end{aligned}
$$

Hence, $\phi^{\mathrm{D}}(\zeta, x)$ and $\pi^{\mathrm{D}}(\zeta, x)$ follow the equation of motion of a non-interacting field, of which the time dependence was discussed previously. Defining

$$
U_{0}^{\mathrm{D}}\left(\zeta^{\prime \prime}, \zeta^{\prime}\right)=\hat{T} e^{-i \int_{\zeta^{\prime}}^{\zeta^{\prime \prime}} H_{0}^{\mathrm{D}}(\eta) e_{\zeta}^{0}(\eta) d \eta}
$$

we have

$$
U_{0}^{\mathrm{D}+}\left(\zeta^{\prime \prime}, \zeta^{\prime}\right)=\hat{T}^{-1} e^{i \int_{\zeta^{\prime}}^{\zeta^{\prime \prime}} H_{0}^{\mathrm{D}}(\eta) e_{\zeta}^{0}(\eta) d \eta}=U_{0}^{\mathrm{D}-1}\left(\zeta^{\prime \prime}, \zeta^{\prime}\right)
$$

Further, by the definition equation (172) of $H_{0}^{\mathrm{D}}(\zeta)$, we have

$$
H_{0}^{\mathrm{D}}(\zeta) e_{\zeta}^{0}(\zeta)=U_{0}^{\mathrm{S}-1}(\zeta, \ell) i \partial_{\zeta} U_{0}^{\mathrm{S}}(\zeta, \ell)
$$


Therefore

$$
i \partial_{\zeta} U_{0}^{S-1}(\zeta, \ell)=-H_{0}^{\mathrm{D}}(\zeta) e_{\zeta}^{0}(\zeta) U_{0}^{S-1}(\zeta, \ell) .
$$

Hence, we have the following relations:

$$
\begin{aligned}
U_{0}^{S-1}(\zeta, \ell) & =\hat{T} e^{i \int_{\ell}^{\zeta} H_{0}^{\mathrm{D}}(\eta) e_{\zeta}^{0}(\eta) d \eta}, \\
U_{0}^{S}(\zeta, \ell) & =\hat{T}^{-1} e^{-i \int_{\ell}^{\zeta} H_{0}^{\mathrm{D}}(\eta) e_{\zeta}^{0}(\eta) d \eta}, \\
\hat{T} e^{-i \int_{\ell}^{\zeta} H_{0}^{\mathrm{S}}(\eta) e_{\zeta}^{0}(\eta) d \eta} & =\hat{T}^{-1} e^{-i \int_{\ell}^{\zeta} H_{0}^{\mathrm{D}}(\eta) e_{\zeta}^{0}(\eta) d \eta,}
\end{aligned}
$$

and we have the inverse transformation

$$
\begin{gathered}
H_{0}^{\mathrm{S}}(\zeta)=\hat{T}^{-1} e^{-i \int_{\ell}^{\zeta} H_{0}^{\mathrm{D}}(\eta) e_{\zeta}^{0}(\eta) d \eta} H_{0}^{\mathrm{D}}(\zeta) \hat{T} e^{i \int_{\ell}^{\zeta} H_{0}^{\mathrm{D}}(\eta) e_{\zeta}^{0}(\eta) d \eta}, \\
|\Psi(\zeta)\rangle^{\mathrm{S}}=\hat{T}^{-1} e^{-i \int_{\ell}^{\zeta} H_{0}^{\mathrm{D}}(\eta) e_{\zeta}^{0}(\eta) d \eta}|\Psi(\zeta)\rangle^{\mathrm{D}} .
\end{gathered}
$$

Suppose that at the initial time $\zeta=\ell$, the system is in the eigen-state $|A ; \ell\rangle_{0}^{\mathrm{S}}$ of $H_{0}^{\mathrm{S}}(\ell)$; then, interaction is turned on adiabatically. At time $\zeta=0$, the interaction is turned off and the state evolves into a state which can be expanded in terms of eigen-states $\left\{|B ; 0\rangle_{0}^{\mathrm{S}}\right\}$ of $H_{0}^{\mathrm{S}}(0)$. The prob of the transition is the square of the amplitude ${ }_{0}^{\mathrm{S}}\left\langle B ; 0\left|U^{\mathrm{S}}(0, \ell)\right| A, \ell\right\rangle_{0}^{\mathrm{S}}[64, \mathrm{p} .323]$, where the Schrödinger picture evolution operator is

$$
U^{S}\left(\zeta_{2}, \zeta_{1}\right)=\hat{T} e^{-i \int_{\zeta_{1}}^{\zeta_{2}} H^{S}(\eta) e_{\zeta}^{0}(\eta) d \eta} .
$$

For a free field, we have

$$
{ }_{0}^{\mathrm{S}}\left\langle B ; 0\left|U^{\mathrm{S}}(0 ; \ell)\right| A, \ell\right\rangle_{0}^{\mathrm{S}}={ }_{0}^{\mathrm{S}}\left\langle B ; 0\left|\hat{T} e^{-i \int_{\ell}^{0} H_{0}^{\mathrm{S}}(\eta) e_{0}^{0}(\eta) d \eta}\right| A, \ell\right\rangle_{0}^{\mathrm{S}},
$$

which was discussed previously. In the interacting case, in terms of the Dirac picture,

$$
\begin{gathered}
|\Psi(\zeta)\rangle^{\mathrm{D}}=\hat{T} e^{-i \int_{\ell}^{\zeta} H_{\mathrm{I}}^{\mathrm{D}}(\eta) e_{\zeta}^{0}(\eta) d \eta}|\Psi(\ell)\rangle^{\mathrm{D}}, \\
|\Psi(\zeta)\rangle^{\mathrm{S}} \\
=\hat{T}^{-1} e^{-i \int_{\ell}^{\zeta} H_{0}^{\mathrm{D}}(\eta) e_{\zeta}^{0}(\eta) d \eta} \hat{T} e^{-i \int_{\ell}^{\zeta} H_{\mathrm{I}}^{\mathrm{D}}(\eta) e_{\zeta}^{0}(\eta) d \eta}|\Psi(\ell)\rangle^{\mathrm{S}} .
\end{gathered}
$$

Hence, we have the expression of the Schrödinger picture evolution operator using only Dirac picture operators:

$$
U^{\mathrm{S}}(\zeta, \ell)=\hat{T}^{-1} e^{-i \int_{\ell}^{\zeta} H_{0}^{\mathrm{D}}(\eta) e_{\zeta}^{0}(\eta) d \eta} \hat{T} e^{-i \int_{\ell}^{\zeta} H_{\mathrm{I}}^{\mathrm{D}}(\eta) e_{\zeta}^{0}(\eta) d \eta},
$$

and $|\Psi(\zeta)\rangle^{S}=U^{S}(\zeta, \ell)|\Psi(\ell)\rangle^{S}$. We have the transition amplitude [64, p.484]

$$
\begin{aligned}
& { }_{0}^{\mathrm{S}}\left\langle B ; 0\left|U^{\mathrm{S}}(0, \ell)\right| A ; \ell\right\rangle_{0}^{\mathrm{S}} \\
= & { }_{0}^{\mathrm{S}}\left\langle B ; 0\left|\hat{T} e^{-i \int_{\ell}^{0} H_{0}^{\mathrm{S}}(\eta) e_{\zeta}^{0}(\eta) d \eta} \hat{T} e^{-i \int_{\ell}^{0} H_{\mathrm{I}}^{\mathrm{D}}(\eta) e_{0}^{0}(\eta) d \eta}\right| A ; \ell\right\rangle_{0}^{\mathrm{S}}
\end{aligned}
$$

This is the basis for perturbational calculations since the second factor can be expanded in terms of powers of coupling constant $\lambda$. In this relation, the dependence of fields in $H_{\mathrm{I}}^{\mathrm{D}}$ on time is the same as in the Heisenberg fields in the noninteracting case while $H_{0}^{\mathrm{S}}$ is the same as in the Schrödinger fields in the noninteracting case. $H_{\mathrm{I}}^{\mathrm{D}}$ is supposed to be expressed in terms of $b_{k}^{\dagger}(\zeta)$ and $b_{k}(\zeta)$ while $H^{\mathrm{S}}$ in terms of $b_{k}^{\mathrm{St}}(\zeta)$ and $b_{\boldsymbol{k}}^{\mathrm{S}}(\zeta)$. These two sets of quasi-particle operators are related to $a_{k}$ and $a_{k}^{\dagger}$.

\section{DISCUSSIONS}

General relativity and QFT are two pillars of modern theoretical physics. As a preamble of a complete unified quantum theory of gravity and matter system, quantum field theories in classical curved spacetimes have long been called for. In this paper, we proposed a generally covariant framework for quantizing the real Klein-Gordon field in de Sitter spacetime. The framework is formulated in a conformal coordinate which is specifically chosen. It can be transformed into other coordinate systems $x^{\prime}$. The fundamental solutions will still be labelled by quantum numbers $\underline{k}$ but the functions will take a more complex appearance depending on the coordinates $x^{\prime}$. The surfaces $\Sigma$ will be defined by functions $\zeta=\zeta\left(x^{\prime}\right)=$ Const. In the new coordinate system $x^{\prime}$, the time dependence becomes actually $\Sigma$ dependence.

It is found that this framework provides many quantum concepts in parallel with the standard quantum field theories in the Minkowski spacetime. The key ingredient for the sake of general covariance is the introduction of vierbein, which furnishes the shift from the local coordinate system to the tangent space. It is well known that concepts such as particle generation and annihilation, particle states, vacuum states, the transition amplitude are very important for quantum theory to explain experiments. The vitality of each physical theory lies in its explanatory power as well as predictive power. The framework proposed in this work has no exception. Primarily, we obtained a reasonable expression of measurable energy and momentum. There are many other topics within this framework to be discussed, topics such as particle generation and perturbative corrections.

Our framework also enjoys three traditional pictures: Heisenberg, Schrödinger, and Dirac in an extended fashion. The Hamiltonians in Heisenberg and Schrödinger pictures are not identical anymore and so the noninteracting Hamiltonians of the Dirac and Schrödinger picture are not equal. Yet, we can nevertheless devise a way to calculate perturbatively the impact of interaction provided that the coupling is weak.

Though de Sitter spacetime is of de Sitter symmetry, it is yet to be investigated whether the symmetry can be realized by quantized fields per se. The generators of de Sitter algebra $\xi_{A B}=z_{A} \partial_{z^{B}}-z_{B} \partial_{z^{A}}$ satisfy

$$
\left[\xi_{A B}, \xi_{M N}\right]=\eta_{B M} \xi_{A N}+\eta_{A N} \xi_{B M}-\eta_{A M} \xi_{B N}-\eta_{B N} \xi_{A M} .
$$

Since at any instant of time $\zeta$, the space is the same as that of the Minkowski spacetime and one can directly find operators

$$
\hat{\xi}_{i j}(\zeta):=\sum_{p} b_{p}^{\dagger}(\zeta)\left(p_{i} \partial_{j}-p_{j} \partial_{i}\right) b_{p}(\zeta)
$$

we realize the spatial part of the de Sitter algebra. We do not know at this moment whether the full de Sitter algebra can be realized by field operators since there might be quantum symmetry breaking.

Lastly, we expect that this framework can be applied to the quantization of spinor fields, and vector fields in de Sitter space 
as well. Yet, for other spacetimes such as Robert-Walker spacetime, the application of this framework might entail some additional steps. The difficulty in the Robert-Walker case is that the known basis solutions $\Pi_{k J}^{( \pm)}(\chi) Y_{J}^{M}(\theta, \varphi), \underline{k}=(k, J, M)$, where $\theta$ and $\varphi$ are angular coordinates [1], are not "plane-waves" anymore, as in the de Sitter spacetime. Hence, extra effort is needed to associate the basis solutions to "free particles". In general, we have to find an appropriate coordinate system in which we can find a complete set of basis solutions, which can be interpreted as (or associated with) "free particles". Once this is achieved, the framework can be transformed to any other coordinate system and thus made generally covariant.

\section{References}

[1] N.D. Birrell \& P.C.W. Davies Quantum Fields in Curved Spacetime (Cambridge University Press, 1982.)

[2] S.A. Fulling Aspects of Quantum Field Theories in Curved Spacetimes (Cambridge University Press,NY,1989)

[3] L.E. Parker \& D.J. Toms Quantum Field Theories in Curved Spacetime (Cambridge University Press,2009)

[4] C. Bär Quantum Field Theories in Curved Spacetimes (Springer,2009)

[5] J.D. Bjorken \& S. D. Drell Relativistic Quantum Fields (McGraw-Hill Book Company, 1965).

[6] J. P. Gazeau \& M.L. Rey. Quantum Field Theory in de Sitter Space hal-00109682,2006

[7] Ta-You Wu, Quantum Mechanics (in Chinese) Vol. I. (Science Publishers,Beijing,1984)

[8] Y.S.Duan \& J.Y. Zhang Acta Physica Sinica (in Chinese) 19 (11):689-704 (1963)

[9] S.S. Feng \& C.G. Huang Int. J. Theor. Phys. 36 (5)(1997):1179-1187.

[10] D. Hochberg \& T.W. Kephart, Phys. Rev. Lett., 66 (1991):2553.

[11] S.S. Feng. Mod. Phys. Lett. B (21)(2001):1385-1393.

[12] M.P. Hobson, G. Efstathiou \& A.N. Lasenby. General Relativity (Cambridge University Press,NY,2006), §5.13.

[13] P.A. M.Dirac, Ann. Math. 36 (3)(1935):657-669.

[14] K. Goto Prog.Theor.Phys. 12 (3) (1954):311-354.

[15] F. Güsey \& T.D. Lee, Proc. N. A.S. 49 (1963):179-186.

[16] C. Fronsdal, Rev. Mod. Phys. 37 (1) (1965):221-224.

[17] K.C. Hannabuss, J. Phys. A 2 (1969):274-277.

[18] C. Fronsdal, Phys. Rev., D10 (2) (1974):589-598.

[19] C. Fronsdal \& R.B. Haugen Phys. Rev., D12 (12) (1975):3810-3818.

[20] C. Fronsdal Phys. Rev., D12 (12) (1975):3819-3830.

[21] T. Brugarino, Ann. Inst. Henri Poincaré, XXXII (1980):277282.

[22] J. Fang \& C. Fronsdal, Phys. Rev., D22 (6) (1980):13611367.

[23] E. Angelopoulos, C. Fronsdal \& D. Sternheimer Phys. Rev., D23 (6) (1981):1278-1289.

[24] E. Joung, J. Mourad \& R. Parentani, Group Theoretical Approach to Quantum Fields in de Sitter Space I, JHEP08(2006)082.

[25] E. Joung, J. Mourad \& R. Parentani, Group Theoretical Approach to Quantum Fields in de Sitter Space II, JHEP09(2007)030.
[26] J. Bros, J.P. Gazeau \& U. Moschella, Phys. Rev. Lett., 73 (13) (1994):1746-1749.

[27] S.A. Pol'shin, Quantization of Fields Over de Sitter Space by the Method of Generalized Coherent States, hepth/0001069v2 \& hep-th/0007091v1 (2000).

[28] N.A. Chernikov \& E.A. Tagirov, Ann. Inst. Henri Poincaré, IX (2) (1968):109-141.

[29] E.A. Tagirov, Ann. of Phys., 76 (1973):561-579.

[30] V. S. Otchik, Class. Quan. Grav. 2 (1985):539-543.

[31] D. V. Gal'tsov \& M.Y. Morozov, Theor. Math. Phys. 77 (2) (1988):190-203.

[32] D. Polarski, Class. Quan. Grav. 6 (1989):717-722.

[33] D. Polarski, Class. Quan. Grav. 6 (1989):893-900.

[34] D. Polarski, Phys. Rev. D 41 (2) (1990):442-450.

[35] D. Polarski, Phys. Rev. D 41 (8) (1990):2519-2514.

[36] V.M Red'kov \& E.M. Ovsiyuk Ricerche. Mat 60 (2011):5788.

[37] O. Nachtmann, Commun. Math. Phys. 6 (1967):1-16.

[38] G. Börner \& H.P. Dürr, IL Nuovo Cimento LXIV (3) (1969):669-713.

[39] T.S. Bunch \& P.C.W. Davies, Proc. R. Soc. Lond. A 360(1978):117-134.

[40] B. Allen \& A. Folacci, Phys. Rev. D 32 (12) (1985):31363149.

[41] E. Mottola, Phys. Rev. D 31 (4) (1985):754-766.

[42] D. Bernard \& A. Folacci, Phys. Rev. D 34 (8) (1986):22862291.

[43] B. Allen \& A. Folacci, Phys. Rev. D 35 (12) (1987):37713778.

[44] I.H. Redmount \& S. Takagi, Phys. Rev. D 37 (6) (1988):1443-1455.

[45] U. Moschella, Ann. Inst. Henri Poincaré, 63 (4) (1995):411426.

[46] J. de Boer, V. Jejjala \& D. Minic, Phys. Rev. D 71 (4) (2005):044013.

[47] I.I. Cotaescu, R. Racoceanu \& C. Crucean Mod. Phys. Lett. A 21 (16) (2006):1313-1318.

[48] A. Casher, P.O. Mazur \& A. J. Staruszkiewicz, Acta Physica Polonica B 42 (6) (2011):1207-1218.

[49] L. D. Landau \& E.M. Lifshitz Mechanics, chapter VII,(Pergamon Press, 1976)

[50] H. Goldstein The Classical Mechanics, 2nd ed. §12.4. (Addison Wesley, Cambridge, Massachusets 1980)

[51] L.C. Crispino, A. Higuchi \& G.E.A. Matsas, Rev. Mod. Phys. 80 (3) (2008):787-838.

[52] E.T. Akhmedov, Int. J. Mod.Phys. D23 (1) (2014):1430001.

[53] V.A. Miransky, Dynamical Symmetry Breaking in Quantum Field Theories. World Scientific, 1993.

[54] N.A. Chernikov, Soviet Phys. JETP 26 (3) (1967):603-608.

[55] H.R. Lewis Jr , Phys. Rev. Lett. 18 (13) (1967):510-512.

[56] H.R. Lewis Jr , J. Math. Phys. 9 (11) (1968):1976-1986.

[57] H.R. Lewis Jr , J. Math. Phys. 10 (8) (1969):1458-1473.

[58] C.J. Eliezer \& A. Gray , SIAM J. Appl. Math. 30 (3) (1976):463-468.

[59] L.F. Landovitz, A.M. Levine \& W.M. Schreiber Phys. Rev. A 20 (3)(1979):1162-1168.

[60] J. Struckmeier \& C. Riedel , Phys. Rev. E 64 (3) (2001):026503.

[61] R. Jackiw Schrödinger Picture for Boson and Fermion Quantum Field Theories. Mathematical Quantum Field Theory and Related Topic (1987):107-134. 
[62] A.L. Fetter \& J.D. Walecka, Quantum Theory of ManyParticle System . Chapter 10, McGraw, 1971.

[63] C. Itzykson \& J.-B. Zuber, Quantum Field Theory . Chapter 4, McGraw-Hill, 1980.

[64] E.Merzbacher, Quantum Mechanics. 3rd ed. (1998). 